$\begin{array}{cl}\begin{array}{c}\text { Revue } \\ \text { de } / \text { histoire } \\ \text { des religions }\end{array} & \text { Revue de l'histoire des religions } \\ & \begin{array}{l}1 \mid 2006 \\ \text { Varia }\end{array}\end{array}$

\title{
Entre la figue et la pomme : l'iconographie romane du fruit défendu
}

Between the Fig and the Apple: The Forbidden Fruit in Romanesque Iconography

Hilário Franco Júnior

\section{(2) OpenEdition}

Journals

Édition électronique

URL : http://journals.openedition.org/rhr/4621

DOI : $10.4000 /$ rhr.4621

ISSN : 2105-2573

Éditeur

Armand Colin

Édition imprimée

Date de publication : 1 mars 2006

Pagination : 29-70

ISBN : 2200-92103-9

ISSN : 0035-1423

Référence électronique

Hilário Franco Júnior, «Entre la figue et la pomme : l'iconographie romane du fruit défendu », Revue de I'histoire des religions [En ligne], 1 | 2006, mis en ligne le 18 janvier 2010, consulté le 30 avril 2019. URL: http://journals.openedition.org/rhr/4621; DOI : 10.4000/rhr.4621 


\section{Entre la figue et la pomme : l'iconographie romane du fruit défendu}

Longtemps accaparées par l'histoire de l'art, les sources iconographiques ont, à peine récemment, commencé à montrer leur potentialité explicative pour d'autres domaines historiques, y compris celui des religions. En effet, elles permettent d'intéressantes réflexions tant sur les questions d'exégèse savante que sur celles de croyances populaires. On essaye ici d'en donner l'exemple à travers l'iconographie romane du fruit défendu. L'examen d'un vaste corpus d'environ trois cents images suggère que le choix de la figue dans ce rôle a eu pour origine un rapport analogique avec le foie, de la même nature que celui existant entre la pomme et le cour. Les relations ainsi mises en lumière dévoilent toute une couche profonde des sensibilités collectives médiévales.

\section{Between the Fig and the Apple : \\ The Forbidden Fruit in Romanesque Iconography}

Long monopolized by art history, iconographic sources have only recently begun to reveal their explicative potential for other fields of history, included the history of religions. They can indeed inspire interesting reflections, whether on questions of learned exegesis or on matters of popular belief. This article seeks to illustrate this, using Romanesque iconography of the forbidden fruit. Studying a vast corpus of some three hundred images, it would appear that when the fig is chosen for this role, there is an analogical relation with the liver, of the same nature as that existing between the apple and the heart. The relations thus brought to light reveal a deep-seated aspect of the medieval collective sensibility. 
Le mythe du péché originel a fortement séduit les chrétiens d'Europe occidentale au Moyen Âge central. Cette force de séduction, il la devait, comme tous les mythes, à sa force explicative, à la réponse qu'il apportait à de nombreuses questions existentielles. D'une part, les hommes du Moyen Âge voyaient en lui le début de toutes les limitations et de toutes les faiblesses de la nature humaine, qu'elles fussent physiques (la petite taille, la menstruation, la calvitie, les maladies, la mort) ou morales (l'orgueil, la malhonnêteté, la concupiscence, l'envie). D'autre part, il expliquait et justifiait un phénomène social essentiel pour l'époque : l'infériorité de la femme. Alors que, pendant les dix premiers siècles du christianisme, la représentation du péché originel s'était concentrée sur les sarcophages (surtout aux $\mathrm{IV}^{\mathrm{e}}$ et $\mathrm{V}^{\mathrm{e}}$ siècles) et sur les croix des cimetières (en particulier aux $\mathrm{IX}^{\mathrm{e}}$ et $\mathrm{X}^{\mathrm{e}}$ siècles), ce qui probablement provenait de la vision pessimiste du christianisme de la période, les choses changèrent après l'an mille.

Parallèlement à la réorganisation sociale connue sous le nom de féodalité, le christianisme occidental déplaça sa visée de Dieu le Père vers Dieu le Fils. L'Incarnation devint l'événement historique central, valorisant ainsi le récit de l'histoire d'Adam, le personnage qui avait rendu nécessaire le sacrifice du Christ. Les thèmes adamiques gagnèrent beaucoup en importance dans la théologie, la littérature et surtout l'iconographie. Selon notre inventaire, forcément toujours provisoire, on connaît en Europe occidentale environ quatre cents images des primi parentes des premiers temps du christianisme jusqu'à la fin du $\mathrm{X}^{\mathrm{e}}$ siècle, et plus de deux mille du XI ${ }^{\mathrm{e}}$ au XIII ${ }^{\mathrm{e}}$ siècle. En ne considérant que les représentations romanes sur les territoires de l'Espagne, de la France et de l'Italie actuelles, nous avons compté plus de huit cents images, dont un tiers concernent le péché originel. Ces images se révèlent une source très riche pour l'historien, car de leur corpus émerge tout un ensemble de valeurs sociales, culturelles, théologiques et psychologiques de l'époque. Même quand s'agit d'un objet d'étude restreint, par exemple le fruit défendu ${ }^{1}$.

1. Pour ce qui concerne les questions méthodologiques de la construction et l'analyse d'un corpus iconographique, de bonnes réflexions sont faites par Jérôme Baschet, "Inventivité et sérialité des images médiévales. Pour une approche iconographique élargie », Annales HSS, 51, 1996, pp. 93-133. 
La consommation du fruit défendu était l'acte fondateur de l'exil humain sur la Terre. Son identification constituait donc un enjeu important pour les hommes du Moyen Âge. On spéculait beaucoup sur sa nature exacte, et l'iconographie ne resta pas à l'écart de ce questionnement. Souvent, on représentait un fruit indéfini, à l'instar du texte biblique ${ }^{2}$. Quelquefois, cette imprécision conduisait à un mélange de caractéristiques : dans les sculptures de la cathédrale de Gérone et de l'église Notre-Dame-du-Port à Clermont-Ferrand (figure 1), les feuilles de l'arbre défendu sont celles du figuier, mais le fruit est le raisin; la célèbre Ève d'Autun cache sa nudité par une feuille de vigne, tout en cueillant une pomme, et le même rapport entre la vigne et la pomme se retrouve à Amandi (Asturies) ; sur un chapiteau de Corbie, Adam semble tenir une pomme, tandis que de l'arbre pendent des grappes de raisin ; sur le chapiteau de Cluny qui représente les fleuves du Paradis, la face orientale représente une vigne, l'occidentale un figuier, la septentrionale un pommier. Comme l'ouest (direction où le soleil se couche, lieu de la mort, « occident » dérivant comme l'on sait d'occidere : «tomber », «mourir») et le nord (l'inconnu, le froid, la source du $\mathrm{Mal}^{3}$ ) avaient des connotations symboliques négatives, l'arbre défendu pourrait être ici le figuier ou le pommier.

Généralement, l'iconographie accomplissait son rôle exégétique et proposait une solution à l'énigme. Tantôt il pouvait s'agir d'un épi de blé, comme sur un chapiteau de San Pedro de Roda (aujourd'hui au musée de Cluny), suivant en cela une interprétation rabbinique ${ }^{4}$, tantôt d'une olive, comme sur la fresque de Saint-Plancard ${ }^{5}$, option

2. Genèse, II, 16-17 ; III, 1-12.

3. Jérémie, I, 14. Jérôme, Expositio quattuor Evangeliorum, Patrologia Latina (PL), t. XXX, col. 549 d-550 a.

4. Midrach Rabba, Genèse, Xv, 7, trad. Bernard Maruani et Albert CohenArazi, Paris, Verdier, 1987, t. I, p. 183 ; Génesis Rabbah I (Génesis 1-11), trad. Luis Vegas Montaner, Estella, Verbo Divino, «Biblioteca Midrasica », 1994, pp. 188-189.

5. D'après l'interprétation de Marcel Durliat, Pyrénées romanes [1969], $2^{e}$ éd., La-Pierre-Qui-Vire, Zodiaque, 1978 (coll. «La Nuit des temps », 30), p. 42. 
toutefois bien plus rare, car fréquemment l'olivier était considéré par les juifs et les chrétiens comme l'arbre de la vie ${ }^{6}$. Plus souvent, on avait pensé à la vigne, aussi bien en Espagne (chapiteau de la cathédrale de Gérone) qu'en France (chapiteaux de Deuil, de SainteGeneviève de Paris, de Saint-Sernin de Toulouse ${ }^{7}$, de Vézelay et de Notre-Dame-du-Port : figure 1). Il faudrait, afin de pouvoir connaître les raisons du choix de la vigne, analyser chaque cas particulier, mais l'on peut penser en général qu'il se fondait sur une tradition hébraïque répandue ${ }^{8}$. Prenons l'exemple de Gérone, ville où l'influence juive semble incontestable. Nous savons qu'au moins depuis 1160 , existait là une "rue des Juifs », et que la pièce la plus importante du trésor de la cathédrale, la célèbre tapisserie de la

6. Vita Adae, 36-42 : «The "Vita Adae" ", éd. J. H. Mozley, The Journal of Theological Studies, 30, 1929, pp. 139-142 (manuscrits anglais) ; «La Vie latine d'Adam et Ève », éd. Jean-Pierre Pettorelli, Archivum latinitatis Medii Aevi, 56, 1998, pp. 55-57 (manuscrits allemands). 2 Henoc 22:8 : Slavonic Apocalypse of Enoch, trad. Francis I. Andersen, in James H. Charlesworth, éd., The Old Testament Pseudepigrapha, Londres, Darton, Longman \& Todd, 1983-1985, 2 vol., t. I, pp. 138-139. L'Évangile de Nicodème, 19, éd. André Vaillant, Genève, Paris, Droz, 1968, pp. 59-61.

7. Dans ce cas, le chapiteau de la porte Miègeville, vers 1100-1118, ne montre pas la scène du péché, mais celle de l'expulsion du Paradis, où le fruit qui se trouve juste derrière Ève et Adam (le couple placé entre Dieu, d'un côté, et un ange de l'autre) est la vigne.

8. Midrach Rabba, Genèse, Xv, 7 et XIX, 5, trad. B. Maruani et A. CohenArazi, op. cit., pp. 184 et 217 ; Génesis Rabbah I, trad. L. Vegas Montaner, op. cit., pp. 190-225. Ethiopic Apocalypse of Enoch, XXXII, 3-6, trad. Ephraim Isaac, in The Old Testament Pseudepigrapha, op. cit., t. I, p. 28. Greek Apocalypse of Baruch, 4-8, trad. Harry E. Gaylord, ibid., t. I, p. 667. Apocalypse of Abraham, XXXIII, 7, trad. Ryszard Rubinkiewicz et Horace G. Lunt, ibid., t. I, p. 700. Au premier siècle de notre ère, les Chapitres d'Éliezer ben Hyrcanos précisent seulement que « Noé a trouvé une vigne avec des grappes de raisin venant du Jardin de l'Éden »: Los Capítulos de Rabbí Eliezer, XXIII, 4, trad. Miguel Pérez Fernandez, Valence, Institución San Jerónimo, 1984, p. 174. Louis Ginzberg pense néanmoins que ce texte ferait probablement allusion à un fragment de l'arbre de la connaissance : Les Légendes des juifs [1909], trad. Gabrielle Sed-Rajna, Paris, Éd. du Cerf, 1997 [coll. « PatrimoinesJudaïsme»], t. I, p. 302, n. 59. Selon le même auteur (ibid., p. 219, n. 70), «l'opinion la plus ancienne et la plus répandue identifie le fruit défendu au raisin, ce qui remonte à une idée mythologique ancienne considérant le vin comme le breuvage des dieux ». 
Création, semble, elle aussi, faire référence au peuple juif ${ }^{9}$. Il est ainsi très probable que le relief du péché originel, de la deuxième moitié du XII ${ }^{\mathrm{e}}$ siècle, qui se trouve dans la galerie occidentale du cloître cathédral, se soit inspiré de récits juifs sur le sujet circulant dans la région.

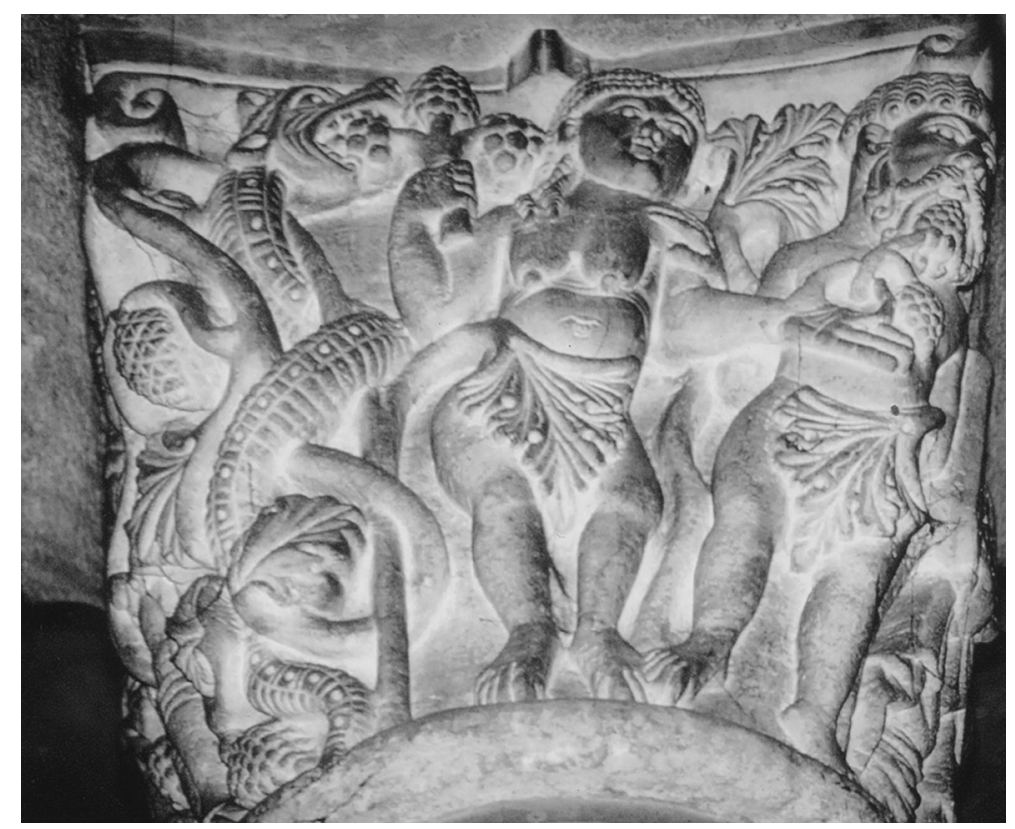

FIGURE 1. Chapiteau du déambulatoire de l'église Notre-Dame-du-Port, Clermont-Ferrand (Auvergne), vers 1120. Photo de l'auteur

D'autre part, il n'y a à notre avis, dans l'art roman italien, aucun exemple connu où la vigne soit représentée comme le fruit défendu de la Bible. Le refus de cette exégèse iconographique fut peut-être

9. David Romano, « Jueus a la Catalunya carolingia i dels primers comtes (876-1100) », in Exposiciò dins la formació de l'Europa medieval, Gérone, Ajuntament de Girona, 1985, pp. 113-119. Hilário Franco Júnior, « Le pouvoir de la parole : Adam et les animaux dans la tapisserie de Gérone », Médiévales, 25, 1993, pp. 123-124. 
une espèce de réaction à la présence dans cette région d'hérétiques de tendance gnostique ${ }^{10}$, qui interprétaient le texte biblique dans ce sens. De fait, dès la deuxième moitié du $\mathrm{II}^{\mathrm{e}}$ siècle, le caractère syncrétique du gnosticisme avait associé les mythes d'Adam et d'Éros : pendant que le démon Jaldabaoth lançait son sperme au sol pour former l'Adam terrestre, son épouse, Pronoia, retirait le sang/ la lumière de l' « homme véritable », l'Adam-Lumière du premier jour de la création, formant ainsi Éros, avec lequel surgit le vin, mélange de feu et de sang qui éveille des désirs voluptueux ${ }^{11}$.

L'indétermination régnait donc, d'une façon générale, quant à l'identité du fruit défendu. Celle-ci peut révéler d'intéressantes transformations socioéconomiques et culturelles, comme on peut le constater à Vézelay. On trouve dans cette basilique deux chapiteaux représentant le péché originel. Sur le plus ancien, daté du $\mathrm{XI}^{\mathrm{e}}$ siècle ${ }^{12}$ et situé dans la nef collatérale nord, Adam et Ève mangent des raisins, tandis que sur celui de la nef centrale, sculpté au deuxième quart $\mathrm{du} \mathrm{XII}^{\mathrm{e}}$ siècle ${ }^{13}$, après l'incendie qui a ravagé une grande partie de l'édifice, la pomme est devenue le fruit du péché. Cela atteste un changement dans le symbolisme du fruit, que l'on peut probablement attribuer à la double valorisation, au

10. Arturo Graf, Il Mito del Paradiso terrestre [1892], rééd., Rome, Edizioni del Graal, 1982, p. 65. Gioacchino Volpe, Movimenti religiosi e sette ereticali nella società medievale italiana : secoli XI-XIV [1922], 4e éd., Florence, Sansoni, 1972, pp. 17-40. Cinzio Violante, La Società milanese nell'età precomunale, $2^{\mathrm{e}}$ éd., Bari, Laterza, 1974, pp. 220-231. L'existence, dans l'Espagne du $\mathrm{VII}^{\mathrm{e}}$ siècle, de prêtres qui offraient à chaque fidèle au moment de l'eucharistie une grappe de raisin pourrait également traduire une réaction à l'idée de la vigne comme le fruit défendu (III e concile de Braga [675], prologue et canon 1: Concílios visigóticos e hispano-romanos, éd. et trad. José Vives, Barcelone, Madrid, CSIC, Instituto Enrique Florez, 1963, pp. 371-373).

11. Michel Tardieu, Trois Mythes gnostiques : Adam, Éros et les animaux d'Égypte dans un écrit de Nag Hammadi (II, 5), Paris, Études augustiniennes, 1974, en particulier les pp. 88-89, 142-144 et 166-169.

12. Paul Deschamps, « Notes sur la sculpture romane en Bourgogne», Gazette des Beaux-Arts, 64, 1922, p. 73.

13. Ibid., p. 76. 
$\mathrm{XII}^{\mathrm{e}}$ siècle, à la fois de l'eucharistie dans tout l'Occident chrétien ${ }^{14}$ et de la viticulture en Bourgogne ${ }^{15}$. Ainsi, l'importance de la vigne pour la religion et pour l'économie locale n'aurait pu s'accommoder avec le caractère négatif du fruit défendu.

$\mathrm{Au} \mathrm{XII}^{\mathrm{e}}$ siècle, la réflexion théologique sur le péché originel était « d'une pensée ardente et variée, d'une grande curiosité d'esprit qui lui fait remuer bien des problèmes, d'une audacieuse liberté parfois, qui lui fait proposer des solutions nouvelles ${ }^{16} »$. En revanche, quand il s'agissait du fruit défendu, elle évitait presque toujours de donner une définition. De son côté, l'iconographie, en fonction du langage employé et du public auquel elle s'adressait, pouvait être plus objective. Mais elle hésitait encore, surtout entre la figue et la pomme. L'héritage classique ne facilitait pas le choix, car il considérait que la figue (liée à Cronos et à Dionysos) et la pomme (associée à Héra et à Aphrodite) étaient tous deux des fruits ambigus, dangereux, sexués, contrairement à l'olive, chaste comme sa créatrice Athéna ${ }^{17}$.

\section{LA FIGUE ET LE FOIE}

De toute façon, la figue apparait dans l'iconographie des trois territoires considérés ici. En Espagne, on la voit sur les fresques de San Justo de Ségovie et Maderuelo (figure 2), dans la peinture sur

14. Joseph de Ghellinck, "L'eucharistie au XII siècle en Occident », in Dictionnaire de théologie catholique, Paris, Letouzey et Ané, 1913, t. V, col. 1233-1302. L'iconographie a aussi été touchée par le phénomène, comme le montre au XIII ${ }^{\mathrm{e}}$ siècle le relief en métal sur la porte de l'église de Sion, en Suisse, où le Crucifié a été représenté comme une grappe de raisin : il est reproduit par Erich Neumann, The Great Mother : An Analysis of the Archetype [1955], trad. Ralph Mannheim, Princeton (N. J.), Princeton University Press, 1972, pl. 114.

15. Roger Dion, Histoire de la vigne et du vin en France des origines au XIX siècle, Paris, chez l'auteur, 1959, pp. 245-247.

16. Auguste Gaudel, «Péché originel », Dictionnaire de théologie catholique, op. cit., t. XII-1, col. 441.

17. Jacques Brosse, Mythologie des arbres, Paris, Plon, 1989, pp. 299-300. C'est cette pureté attribuée à l'olive qui faisait de l'olivier l'arbre de la vie par excellence, comme on a vu supra, n. 5 . 


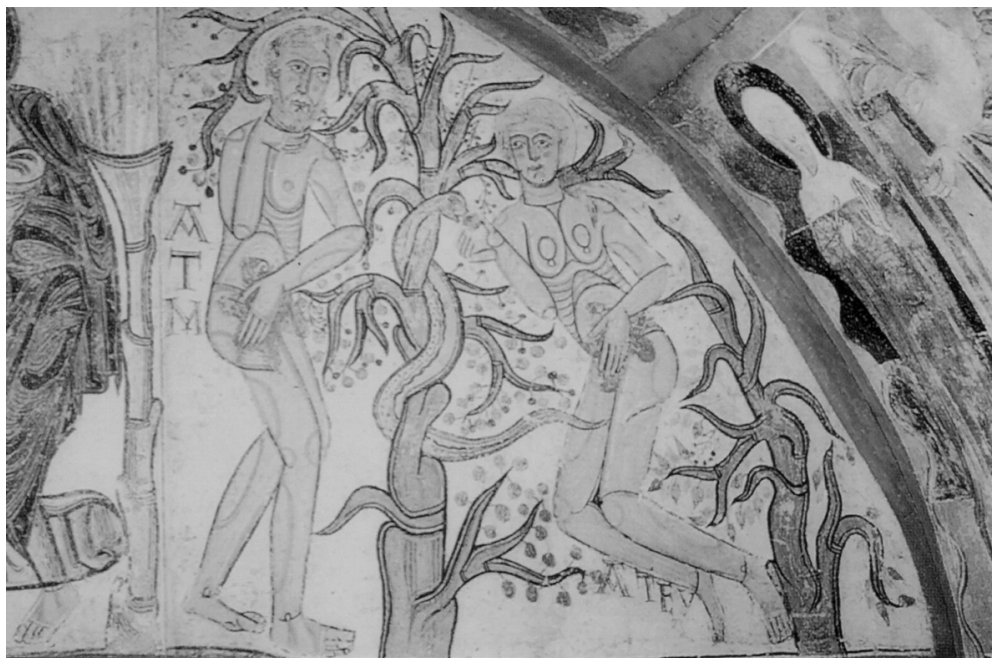

Figure 2. Fresque sur le mur occidental de la chapelle de la Vera Cruz de Maderuelo (Ségovie, actuellement au musée du Prado), Castille, vers 1125. Photo du musée

bois de Sagàs et dans les sculptures de Barrio Santa Maria et de Butrera. En France, elle est figurée sur les chapiteaux de Barret, de Lavaudieu et de la Sauve-Majeure, et sur l'archivolte de SaintMartin de Besse. En Italie, elle est présente dans les mosaïques d'Otrante, de Monreale et de Trani, dans les sculptures en pierre et en métal de San Zeno à Vérone, ainsi que dans l'enluminure de l'Exultet 3 de Troia (figure 5). À l'abbatiale de Sainte-Marie de Cruas (Ardèche), la mosaïque du pavement du chœur, datant du premier quart du XII ${ }^{\mathrm{e}}$ siècle, représente sous les mains de Dieu le patriarche Enoch et le prophète Élie, ce dernier étant placé à la gauche de l'observateur et donc au-dessous de la droite divine. Entre les deux hommes apparaissent deux arbres stylisés avec des inscriptions, Lignum sur celui du côté d'Élie, et Ficus sur celui du côté d'Enoch, ce qui a amené à identifier le premier comme l'arbre de la vie et le deuxième comme celui de la connaissance du bien et du mal ${ }^{18}$.

18. Robert Saint-Jean et Jean Nougaret, Vivarais-Gévaudan romans, La Pierre-Qui-Vire, Zodiaque, 1991 (coll. « La Nuit des temps », 75), pp. 157-158. 
Cette solution iconographique au problème de l'identification du fruit est suggérée par le récit même de la Genèse, selon lequel peu après avoir commis le péché, honteux, Adam et Ève se couvrirent de «feuilles de figuier ${ }^{19}$ ». Un autre texte biblique renforce cette idée : présentant cet arbre comme un lieu de méditation, il l'associe à la connaissance et suggère ainsi qu'il s'agit de l'arbre défendu ${ }^{20}$. Une telle interprétation se fondait encore sur le fait que le Christ avait maudit le figuier, l'arbre qui, selon des traditions populaires, aurait été celui sous lequel Jean-Baptiste fut décapité et à la branche duquel Judas se pendit ${ }^{21}$, tel que le figure une enluminure allemande du IX ${ }^{\mathrm{e}}$ siècle $^{22}$. Cette association entre l'arbre du péché d'Adam et l'arbre de la punition du péché de Judas - tous les deux des traîtres au Seigneur - se retrouve à la cathédrale d'Autun : une sculpture y montre Judas pendu à un arbre similaire à celui qui couvre le sexe d'Ève dans la scène célèbre du linteau du portail nord, actuellement déposé au musée Rolin.

À partir de ces éléments, on a plusieurs fois considéré la figue comme étant le fruit défendu de l'Éden. Tout au moins, c'est ce que fit un commentaire rabbinique ${ }^{23}$. La même démarche apparaît dans quelques apocryphes chrétiens. La version grecque de la Vita Adae et Evae, par exemple, dit qu'immédiatement après la faute, toutes les feuilles des arbres du Paradis tombèrent, à l'exception de celles,

19. Genèse, III, 7.

20. Jean, I, 48. Ce rapport entre la figue et la connaissance remontait au paganisme classique : Platon, par exemple, appelait ce fruit « l'ami des philosophes », selon Éloïse Mozzani, Le Livre des superstitions : mythes, croyances et légendes, Paris, Robert Laffont, 1995, p. 746.

21. Matthieu, XXI, 19. Paul Sébillot, Le Folklore de France, t. VI : La Flore [1906], rééd., Paris, Imago, 1985, p. 21. É. Mozzani, Le Livre des superstitions, loc. cit.

22. Psautier de Stuttgart, vers 810 (Stuttgart, Württembergische Landesbibliothek, Cod. Bibl. $172^{\circ} 23$, fol. 8).

23. Midrach Rabba, Genèse Xv, 7, trad. B. Maruani et A. Cohen-Arazi, op. cit., pp. 185 ; Génesis Rabbah I, trad. L. Vegas Montaner, op. cit., pp. 190191. 
affirme Ève, « de la plante que j'ai mangée », le figuier ${ }^{24}$. Dans un autre texte, c'est Adam qui, répondant à une question de son fils Seth, affirme que le fruit défendu était une figue ${ }^{25}$. Un autre texte suggère la même chose, en disant qu'après l'expulsion du Paradis, les primi parentes se nourrirent d'abord de figues envoyées par Dieu, des fruits avec saveur «de pain et de sang ${ }^{26} »$. Quelques auteurs chrétiens orientaux ont été dans le même sens, par exemple Théodoret de $\mathrm{Cyr}^{27}$ (vers 393-vers 458). Parmi les théologiens occidentaux qui glosèrent le texte biblique dans le même sens, on trouve Tertullien, Hugues de Saint-Victor et Pierre Comestor ${ }^{28}$. Les exemples littéraires sont rares, mais existent, même à une époque plus avancée, comme chez Gottfried de Strasbourg ${ }^{29}$.

24. Life of Adam and Eve (Apocalypse), xx, 4-5, trad. M. D. Johnson, in The Old Testament Pseudepigrapha, op. cit., t. II, p. 281 ; Apocalisse di Mosè, trad. Liliana Rosso Ubigli, in Paolo Sacchi, dir., Apocrifi dell'Antico Testamento, Turin, UTET, 1989, t. II, p. 429 ; Vida de Adán y Eva (Apocalipsis de Moises), trad. Natalio Fernández Marcos, in Alejandro Diez Macho, dir., Apocrifos del Antiguo Testamento, Madrid, Cristiandad, 1982, t. II, p. 330.

25. Testament of Adam 3c, trad. Stephen E. Robinson, in The Old Testament Pseudepigrapha, op. cit., t. I, p. 994 ; Testamento de Adán III, 4 (R II), trad. F. J. Martínez Fernández, in Apocrifos del Antiguo Testamento, op. cit., t. V, p. 433.

26. Il Combattimento di Adamo, 40, éd. et trad. A. Battista et B. Bagatti, Jérusalem, Franciscan Printing Press, 1982 (« Studium Biblicum Franciscanum, Collectio Minor », 29), p. 110.

27. Théodoret de Cyr, Quaestiones in Genesim, II, 28, Patrologia Graeca $(P G)$, t. LXXX, col. 125 c.

28. Tertullien, Adversus Marcionem, I, 2, 2, éd. Ernst Kroymann, Turnhout, Brepols, 1954 («Corpus christianorum. Series latina », 1), p. 443. Hugues de Saint-Victor, Adnotationes elucidatoriae in Pentateuchon, Patrologia Latina $(P L)$, t. CLXXV, col. 42 a-b. Pierre Comestor, Historia scholastica, 23, PL, t. CXCVIII, col. 1073 b-c. Même à la fin du Moyen Âge, quelques auteurs pensaient encore de cette façon: Maître Eckhart, Commentaire de la Genèse, 97 et 205, éd. et trad. Fernand Brunner et al., Paris, Éd. du Cerf, 1984 (L'Euvre latine de Maître Eckhart, 1), pp. 360 et 518.

29. Das Tristan-Epos Gottfrieds von Strassburg, v. 17944, éd. Wolfgang SPIEWOK, Berlin, Akademie-Verlag, 1989 (« Deutsche Texte des Mittelalters », 75), p. 251. 
De toute manière, l'exégèse qui voyait la figue comme le fruit défendu avait des traits nettement archaïsants ${ }^{30}$. En effet, malgré son importance pour l'alimentation des régions méditerranéennes, le figuier était considéré comme un arbre impur par les Grecs et les Romains $^{31}$. Or, au moins depuis le $\mathrm{XII}^{\mathrm{e}}$ siècle, on croyait que la croix du Christ avait été fabriquée avec le bois de l'arbre défendu ${ }^{32}$; cela renforçait l'idée qu'il s'agissait du figuier, considéré par les Anciens comme l'arbre de Dionysos, divinité qui présentait de claires analogies avec le Christ (tous les deux sont les fils de la divinité et d'une mortelle ; les deux meurent et ressuscitent ; le sang-vin des deux est consommé rituellement par les fidèles ; les deux descendent en Enfer).

Plus probablement, l'identification de la figue avec le fruit défendu était associée au symbolisme du foie. Pour les Hébreux, cet organe avait des connotations sacrées, d'où la prescription de brûler sur l'autel, lors des sacrifices à Yaveh, le foie et les reins de l'animal, avec la graisse qui les entoure ${ }^{33}$; d'où aussi la croyance qu'en brûlant le foie d'un poisson, la fumée avait le pouvoir d'expulser le démon ${ }^{34}$. Pour les Grecs, cet organe était le centre vital du corps humain. C'est précisément ce que rappelle, à titre d'exemple, le mythe de

30. Beryl Smalley, « Andrew of Saint-Victor, abbot of Wigmore : a twelfthcentury hebraist », Recherches de théologie ancienne et médiévale, 10, 1938, pp. 358-373. Id., The Study of the Bible in the Middle Ages [1952], 2e éd., Oxford, Basil Blackwell, 1983, pp. 149-172 et 179-180. Esra Shereshevsky, «Hebrew Traditions in Peter Comestor's Historia Scholastica », The Jewish Quartely Review, 59, 1968-1969, pp. 268-289.

31. J. Brosse, Mythologie des arbres, op. cit., pp. 285-286.

32. Jean Beleth, Summa de ecclesiasticis officiis, 125, éd. Herbert Douteil, Turnhout, Brepols, 1976 ( « Corpus christianorum. Continuatio medievalis » [CCCM], 41 A), pp. 239-241 ; Gervais de Tilbury, Le Livre des merveilles : divertissement pour un empereur, 105, trad. Annie Duchesne, Paris, Les Belles Lettres, 1992 (coll. «La Roue à livres »), p. 129. Au XIII ${ }^{\mathrm{e}}$ siècle, le thème apparaît dans quelques textes bien connus, tels La Queste del Saint Graal (éd. Albert Pauphilet, Paris, Honoré Champion, 1980, p. 210 et suiv.) et la Légende dorée de Jacques de Voragine (Legenda aurea, vulgo Historia Lombardica dicta, LXVIII, éd. Theodor Graesse [1846], rééd., Osnabrück, Otto Zeller, 1969, pp. 303-304).

33. Exode, XXIX, 13, 22 ; Lévitique, III, 4, 10, 15 ; IV, 9 ; VII, 4 ; VIII, 16, 25 ; IX, 10, 19.

34. Tobie, VI, 7. 
Prométhée, dont la punition pour avoir donné le feu aux hommes fut d'avoir son « foie immortel » (symbole de lui-même) quotidiennement dévoré par un aigle (symbole du monde olympien ${ }^{35}$ ). Dans le mythe d'Éros, ce dieu, pour séduire les humains, visait leur foie de ses flèches ${ }^{36}$, d'où la conviction d'Horace que c'est le foie qui fait aimer ${ }^{37}$. De son côté, Platon établit un rapport étroit entre âme et foie, voyant en celui-ci une création divine qui permet à celle-là d'exercer l'activité de divination ${ }^{38}$. Ce n'est que dans les versions tardives du mythe d'Éros (les peintures murales de Pompéi, par exemple), expressions d'un changement culturel plus vaste, que les flèches touchent le cœur ${ }^{39}$. Encore au XIII ${ }^{\mathrm{e}}$ siècle, le foie n'avait pas perdu définitivement sa signification antérieure : la somme hagiographique la plus connue de l'époque, bien qu'elle attribuât presque toujours le rôle principal au cœur, faisait encore du foie, au moins dans un passage, le centre vital de l'être humain ${ }^{40}$.

35. Hésiode, Théogonie, v. 524, éd et trad. Paul Mazon, $13^{\mathrm{e}}$ tirage, Paris, Les Belles Lettres, 1996 (Coll. des Universités de France), p. 51.

36. Anacréon, «Fragment 33 », vv. 28, 32, in Carmina Anacreontea, éd. Martin L. West, Leipzig, B. G. Teubner, 1984, p. 25.

37. Horace, Odes, IV, 1, 12, éd. et trad. François Villeneuve, Paris, Les Belles Lettres, 1927 (Coll. des Universités de France), p. 152.

38. Platon, Timée, 71 a, d, éd et trad. Albert Rivaud, Paris, Les Belles Lettres, 1985 (Coll. des Universités de France), p. 198.

39. À l'époque romane, il y a eu au moins une allusion faite au Cupidon latin (appelé seulement Amors) fléchant le cœur : Chrétien de Troyes, Cligès, v. 455, trad. Alexandre Micha, Paris, Honoré Champion, 1982 (Traductions des Classiques français du Moyen Âge [CFMA]), p. 14. Un recueil médiéval de mythologie classique, écrit entre 875 et 1075, dit que pour punir Prométhée les dieux envoyèrent une aigle lui ronger le cœur (et non le foie, comme l'affirmait Hésiode) : Premier Mythographe du Vatican, I, 1, 3, éd. Nevio Zorzetti, trad. Jacques Berlioz, Paris, Les Belles Lettres, 1995 (Coll. des Universités de France), p. 2. La transposition de la charge symbolique du foie au cœur devint si enracinée que des érudits modernes ont prit plus d'une fois un mot pour l'autre, comme, par exemple, le traducteur d'Horace cité supra n. 36, ou celui d'Anacréon (Odes, trad. Frédéric Matthews, Paris, Presses Universitaires, 1927, p. 91).

40. Jacques de Voragine, Legenda aurea, XXV, op. cit., p. 120. Au $\mathrm{XVII}^{\mathrm{e}}$ siècle encore, Cervantes utilise l'expression " enamorado hasta los hígados » : Don Quijote de la Mancha, I, 26, éd. Francisco Rodríguez Marín, Madrid, Atlas, 1947, t. II, p. 297. 
Cette interprétation est demeurée inscrite dans les langues romanes alors en formation, comme le montre le sens figuré de " courage » que le mot garde en italien, en castillan, en catalan et en galicien (ici au pluriel, foies), ainsi que le sens d' « intime », «profond», « entrailles », "viscéral », présent dans le mot portugais figadal (dérivé de fígado, «foie »). En français, des significations similaires apparaissent par inversion, l'absence figurée du foie signifiant la peur : du mot classique ficatum, le latin populaire avait fait feticare, « avoir l'aspect du foie », duquel sortit au XII ${ }^{\mathrm{e}}$ siècle fegier, devenu vers 1225 figer, au sens de « coaguler [le sang] »; en 1592, alors que depuis longtemps le cœur avait remplacé le foie dans le rôle d'organe central, ce mot gagnerait l'acception de « rendre immobile ${ }^{41} »$.

Dans les langues germaniques aussi, le mot a conservé cette double acception de partie du corps humain et de valeur morale positive. C'est le cas de l'anglais liver (attesté depuis la fin du $\mathrm{IX}^{\mathrm{e}}$ siècle), dont la première graphie, lifer, ne laisse pas de doute quant à sa dérivation de life, « vie ». D'où le fait que liver signifie, outre «foie », « vivant» (et, par extension, « habitant»). Comme le foie a une couleur foncée, on parle du white liver comme caractéristique du lâche ${ }^{42}$. En plus d'indiquer le foie, le mot allemand Leber a également conservé sa charge positive, avec l'acception de « courage », « caractère », « sincérité », comme dans l'expression frei von der Leber reden ("parler en toute franchise »). La même racine et le même sens profond apparaissent dans Lebewesen, « être vivant ».

En latin classique, « foie » était dit iecur jusqu'à l'apparition, au premier siècle, du terme ficatum («foie plein de figue ») : basé sur le modèle grec (sykon, d'où heper sykoton), ce dernier désignait le résultat d'une ancienne pratique, l'engraissement de certaines volailles avec des figues. Les Égyptiens auraient été les premiers à

41. Pierre Guiraud, Dictionnaire des étymologies obscures, Paris, Payot, 1982, s.v. Alain Rey, dir., Dictionnaire historique de la langue française, Paris, Le Robert, 1992, t. I, s.v. Dans le même sens, au XIXe siècle ont surgi des expressions comme « avoir les foies blancs », « se ronger les foies », « avoir les foies ».

42. The Oxford English Dictionary, Oxford, Clarendon Press, 1933, t. VI, pp. 360-361. 
remarquer, trois mille ans avant notre ère, que les oies et les canards passaient, avant leur migration, par une période d'alimentation excessive, pendant laquelle ils constituaient une réserve énergétique pour leur voyage, ce qui rendait leur foie particulièrement savoureux. Pour reproduire artificiellement le phénomène, les Égyptiens en seraient venus à gaver les volailles avec des figues sèches, pratique qu'auraient connue puis répandue les Hébreux. Terme médical ${ }^{43}$ ou culinaire $^{44}$ à l'origine, ficatum en vint à signifier « foie d'animal » et, dès le III ${ }^{\mathrm{e}}$ siècle, «foie » en général. C'est de ce mot latin un peu christianisé qu'ont dérivé, quelques siècles plus tard, ses correspondants dans les divers parlers romans : le français «foie », l'italien fegato, le castillan hígado, le portugais figado, le roumain ficat, le provençal et le catalan fetge, le gascon hitge, le picard fie, le wallon féte, le vénitien figa, le frioulan fiyat, le piémontais fidegh, l'abruzzien feteche, le campanien figau, le sarde figadu, le sicilien ficatu, le galicien figado, l'asturien fegadu. Aucun de ces mots n'est en relation avec le péché originel. Il en subsiste toutefois un vestige dans l'italien vulgaire : fica (« vagin » et, par métonymie, « femme attrayante »), sens dérivé de fica, forme vulgaire du latin classique ficus ( « figue »); ce mot, déjà féminin dans la langue classique, semble marquer dans

43. Selon Innocenzo Mazzini, ce fut au $\mathrm{II}^{\mathrm{e}}$ siècle, avec Galien (De alimentarum facultatibus), que l'on attribua pour la première fois un caractère guérisseur à certains mets, comme le foie des animaux alimentés de figues ( "L'alimentation et la médecine dans le monde antique », in Jean-Louis Flandrin et Massimo Montanari, dir., Histoire de l'alimentation, Paris, Fayard, 1996, p. 262). Cette idée a traversé tout le Moyen Âge, où ce plat n'était servi que comme médicament, d'après Silvano Serventi, La Grande Histoire du foie gras, Paris, Flammarion, 1993, p. 73. Dans la longue durée historique, le rapport figue-foie-médicament apparaît dans le folklore brésilien avec le personnage du papa-figo, qui tue des enfants pour en manger le foie, espérant par-là être guéri de la lèpre, selon Gilberto Freyre, Casa-Grande e senzala [1933], $31^{\mathrm{e}}$ éd., Rio de Janeiro, Record, 1996, p. 328 ; Luis da Camara Cascudo, Dicionário do folclore brasileiro [1954], 6e éd., Belo-Horizonte, São Paulo, Itatiaia, Edusp, 1988, pp. 576-577.

44. Alfred Ernout et Antoine Meillet, Dictionnaire étymologique de la langue latine [1931], 4e éd., Paris, Klincksieck, 1959, pp. 232 et 307. Veiko Väänänen, Introduction au latin vulgaire, Paris, Klincksieck, 1967, p. 84. 
la physiologie féminine la conséquence du péché originel ; il a pour correspondant masculin le pomo d'Adamo, le cartilage thyroïde qui rappelle le fruit défendu resté bloqué dans la gorge du premier homme puis de ses descendants.

\section{LA POMME ET LE CCEUR}

Toutefois, l'iconographie romane a utilisé davantage la pomme comme le fruit défendu. La longue liste de ces images dans les trois pays qui nous intéressent représente une partie significative de notre corpus. Parmi elles, on peut citer, en Espagne, Amandi, Añes, Avilés, la Bible de Burgos, la Bible de San Isidoro, Covet, Estany, Estibaliz, Frómista, Loarre, Mahamud, Peralada (figure 6), Porqueras, Rebolledo de la Torre, San Pablo del Campo, Sangüesa, Santillana del Mar et Uncastillo. En France, Airvault, Andlau, Arles, Aulnay, la Bible de Corbie, la Bible de Marchiennes, la Bible de Souvigny, Cahors, Chalon-sur-Saône, Chauvigny (figure 3), Cluny, Courpiac, Esclottes, Guarbecque, Hastingues-Arthous, l'Hortus Deliciarum, Lescure, Mauriac (en Auvergne), Melay, Moirax, Montpezat, Neuillyen-Donjon, Nîmes, Poitiers (église Sainte-Radegonde), Provins, Saint-Benoît-sur-Loire, Saint-Gaudens, la Sauve-Majeure, Targon, Tavant, Thuret, Toirac, Varax, Verdun et Vézelay. En Italie, Galliano, Modène (figure 4), Parme, Pise, Sant'Angelo in Formis et Sovana.

Au long des siècles suivants, la pomme se fit constamment présente dans l'iconographie du péché originel ${ }^{45}$. Aussi fut-elle fréquemment utilisée comme le fruit défendu dans la littérature, notamment, au XII ${ }^{\mathrm{e}}$ siècle, par Marie de France ${ }^{46}$, au XIII ${ }^{\mathrm{e}}$ siècle par

45. À titre d'illustration, rappelons que la pomme apparaît, à l'époque gothique, dans presque toutes les représentations. Dans l'art de la Renaissance, elle a été choisie, entre autres, par Tintoret, Dürer, Lucas Cranach, Lucas van Leyden, Jan Sadeler, Jean de Gourmont. Dans l'art classique, par Rembrandt, Simone Cantarini et Laurent Cars. Dans l'art romantique, par Hyacinthe-JeanBaptiste Aubry-Lecomte et Joseph-Urbain Melin.

46. Marie de France, Yonec, v. 152, in Les Lais de Marie de France, éd. Jean Rychner, Paris, Honoré Champion, 1978 (CFMA), p. 107. 


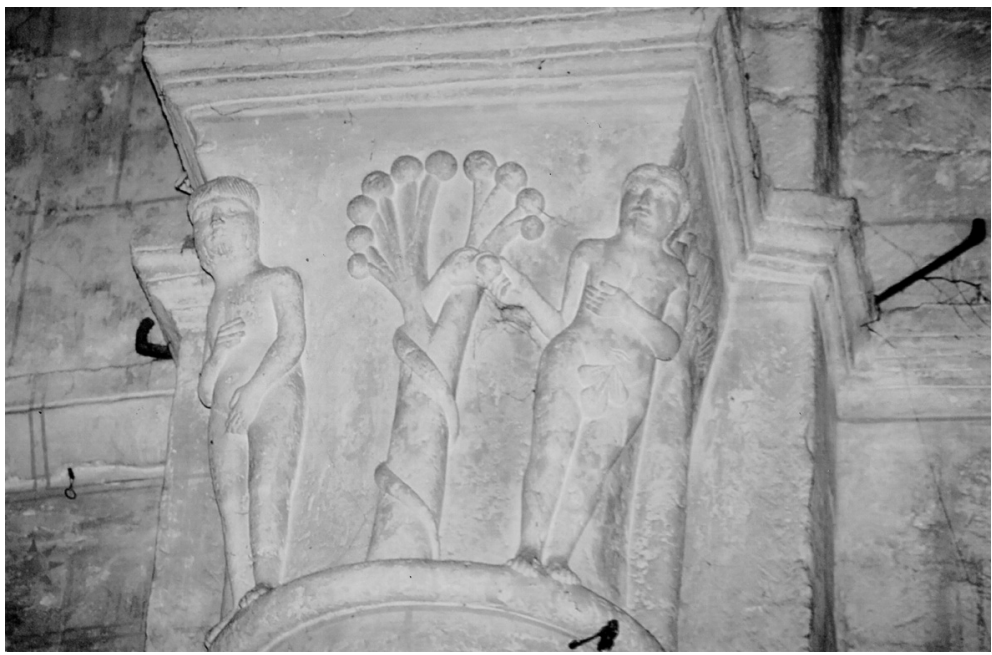

Figure 3. Chapiteau de l'entrée du chœur de l'église

Notre-Dame, à Chauvigny (Poitou), XII ${ }^{\mathrm{e}}$ siècle.

Photo de l'auteur

Robert de Boron ${ }^{47}$, au XV siècle par Sebastian Brandt ${ }^{48}$. Dans la parémiologie, ceci semble être le sens d'un proverbe du début du XIII ${ }^{\mathrm{e}}$ siècle selon lequel « mieux vaut pomme donnée que mangée ${ }^{49}$ ». Dans l'hagiographie, la pomme est le fruit défendu, par exemple, dans les Cantigas de Santa María ${ }^{50}$. Nous en avons également un cas intéressant dans le bréviaire : le Je vous salue Marie - apparu au $\mathrm{XII}^{\mathrm{e}}$ siècle, à partir d'un passage néo-testamentaire ${ }^{51}$ - fait référence uniquement à un «fruit», mais un commentateur anonyme de la

47. Le Roman du Graal : manuscrit de Modène, éd. Bernard Cerquiglini, Paris, Union générale d'édition, 1981 (coll. «10-18. Bibliothèque médiévale »), pp. 28 et 50 .

48. Sebastian Brandt, La Nef des fous [Das Narrenschiff], 12, adapt. Madeleine Horst, Strasbourg, Éd. de la Nuée bleue, 1989, p. 46.

49. Joseph Morawski, éd., Proverbes français antérieurs au XVe siècle, Paris, Édouard Champion, 1925 (CFMA), p. 47 : Meuz valt pume duné que mangé.

50. Alphonse X de Castille, Cantigas de Santa María, 353, v. 11, éd. Walter Mettmann, Madrid, Castalia, 1989, t. III, p. 214.

51. Luc, I, 28, 42. Henri Leclercq, " Marie, mère de Dieu », in Dictionnaire d'archéologie chrétienne et de liturgie, Paris, Letouzey et Ané, 1932, t. X-2, col. 2043-2062. 
France du Nord précise, à la fin du XIII ${ }^{\mathrm{e}}$ siècle ou au début du XIV e siècle, qu'il s'agit du « fruit du pommier ${ }^{52}$ ». Ancrée dans les imaginaires occidentaux depuis lors, la pomme s'est substitué à la figue même chez les érudits modernes, de façon complémentaire au processus culturel qui les a conduits à voir le cœur où il y avait le foie ${ }^{53}$.

Mais les motifs de ce choix presque unanime ne sont pas clairs. On peut évoquer la présence plus ou moins répandue de la pomme dans toute l'Europe occidentale. On peut considérer l'ancien symbolisme celte de la pomme comme le fruit de la connaissance. On peut rappeler son capital symbolique, signe de pouvoir, de richesse, de mensonge, de luxure, de discorde et de transgression ${ }^{54}$. On peut penser que, tout comme le jardin des Hespérides rappelle le jardin de l'Éden (tous les deux abritant un serpent défenseur de l'arbre sacré), le pommier «aux fruits d'or» du mythe grec a contaminé l'interprétation médiévale du récit biblique. On peut argumenter de l'ancienne association existant entre cet arbre et l'Éden, qui fit appeler en hébreu la caroube «pomme du Paradis ${ }^{55}$ ». On peut considérer l'autorité de saint Augustin, qui, bien qu' avec hésitation, acceptait la possibilité pour la pomme d'avoir été le fruit du péché, peut-être influencé par l'existence de trente variétés différentes de pommes dans le monde romain de son époque ${ }^{56}$. On peut surtout se

52. Munich, Bayerische Staatsbibliothek, Cod. Gall. 34, traduit par Geneviève Hasenohr, dans Nicole Bériou, Jacques Berlioz et Jean Longère, dir., Prier au Moyen Âge : pratiques et expériences, $V^{e}-X V^{e}$ siècles, Turnhout, Brepols, 1991, p. 282.

53. Voir supra n. 38. À propos de la pomme, un exemple parmi d'autres, figure dans une traduction de Gottfried de Strasbourg (Bernd Dietz, Tristán e Isolda, Madrid, Nacional, 1982, p. 339) qui rend le haut-moyen allemand vîge par manzana.

54. Michel Pastoureau, «Bonum, malum, pomum. Une histoire symbolique de la pomme », in id., dir., L'Arbre : histoire naturelle et symbolique de l'arbre, du bois et du fruit au Moyen Âge, Paris, Le Léopard d'or, 1993 (« Les Cahiers du Léopard d'or », 2), pp. 155-159.

55. L. Ginzberg, Les Légendes des juifs, op. cit., p. 219, n. 70.

56. Augustin, La Genèse au sens littéral en douze livres [De Genesi ad litteram in duodecim], VIII, VI, 12, éd. Joseph Zycha, trad. Paul Agaësse et Aimé Solignac, Paris, Desclée de Brouwer, 1972 ("Euvres de saint Augustin », 49), t. II, pp. 28-31. J. Brosse, Mythologie des arbres, op. cit., p. 292. 
demander si dans l'étymologie populaire médiévale il n'y aurait pas une certaine confusion entre les mots malum, « mal », et malum, « pomme », ainsi qu'entre malus, « méchant », et malus, « pommier », identités phonétiques qui auraient eu des implications sémantiques indiquant le caractère maléfique du fruit ${ }^{57}$.

La popularité croissante de la pomme dans ce rôle était peut-être aussi liée à sa forme arrondie et à sa couleur rouge, qui la rapprochait du cœur, organe où le christianisme et sa mystique liée au sang versé du Christ voyaient le centre de l'être humain. Les précédents en ce sens étaient forts, car il semble que l'hésitation quant à l'identité du fruit défendu ait reflété celle, plus ancienne, quant à l'organe central du corps dans les diverses cultures qui, de façon plus ou moins directe, ont fourni des éléments à la culture chrétienne médiévale. Alors que les Égyptiens voyaient dans le cœur le centre de l'être humain ${ }^{58}$, les Hébreux attribuaient des pouvoirs sacrés au foie, tout en considérant le cœur comme le siège des sentiments et de la sagesse, et la source de la vie ${ }^{59}$. Les deux organes se disputaient le rôle de principe de la vie chez les Babyloniens ${ }^{60}$ et les Grecs ${ }^{61}$.

57. Parmi les transformations qui affectent le monde romain à partir de la crise du $\mathrm{III}^{\mathrm{e}}$ siècle, on note un changement dans la prononciation du latin, avec la disparition progressive de la distinction entre les syllabes longues et les syllabes brèves (V. Väänänen, Introduction, op. cit., pp. 29-74). Cela permettait des jeux de mots comme le proverbe Mala mali malo mala contulit omnia mundo, c'est-à-dire « la mandibule a apporté tous les malheurs du monde par la pomme du Malin » (Hans Walther, Proverbia sententiaeque latinitatis medii aevi. Lateinische Sprichwörter und Sentenzen des Mittelalters, Göttingen, Vandenhoeck und Ruprecht, 1963, t. I, n ${ }^{\circ}$ 14301) et sa variante postérieure, Mala mali malo meruit mala maxima mundo (ibid., t. II-8, 1983, no $38062 \mathrm{c1}$ ).

58. The Book of the Dead, éd. et trad. E. A. Wallis Budge, introduction et chapitres XVII, XXI, XXVI, CXLIV, CXLV, CXLVIII, rééd., Harmondesworth, Penguin, 1989, pp. 22-34, 107, 131, 138-139, 444-445, 449-460 et 483.

59. Cas, parmi beaucoup d'autres, de Genèse, XX, 5 ; Job, IX, 4 ; Proverbes, IV, 23.

60. Alexandre Piankoff, Le "Cour» dans les textes égyptiens depuis l'Ancien jusqu'à la fin du Nouvel Empire, Paris, PUF, 1930, p. 52.

61. Comme on le sait, dans la mythologie, le foie est l'élément central dans l'histoire de Prométhée, tandis que dans celle de Dionysos, c'est le cœur. Dans le domaine de la médecine, le rôle principal est joué, pour plusieurs auteurs, par le foie et, pour d'autres, par le cœur, surtout à partir d'Aristote (C. S. Harris, The Heart and the Vascular System in Ancient Greek Medecine, Oxford, Clarendon Press, 1973). 
Au III ${ }^{\mathrm{e}}$ siècle avant J.-C., l'école médicale d'Alexandrie établit le modèle physiologique qui allait prédominer tout au long des deux millénaires suivants : au cerveau étaient attribués la sensibilité, le mouvement et les fonctions neurologiques, au cœur, l'enthousiasme et l'esprit vital ${ }^{62}$.

Isidore de Séville affirmait que « dans le cœur se trouve l'origine de la science, [car] on connaît les choses avec le cœur et on les aime avec le foie ${ }^{63} »$. Partageant son point de vue, plus de cinq siècles plus tard, Hildegarde de Bingen pensait que la propriété du cœur était la connaissance et celle du foie la sensibilité ${ }^{64}$. Pour elle, le cœur était le point de liaison entre le corps et l'âme, entre le terrestre et le divin ; il était "presque l'essence du corps [puisqu'il] le régit », la résidence de l'âme ${ }^{65}$. Aussi n'est-ce pas un hasard si elle imaginait le fruit défendu comme une pomme ${ }^{66}$. Pour saint Bernard, le cœur était le siège de la foi ${ }^{67}$. Pour son adversaire, Pierre Abélard, quand Dieu veut examiner les sentiments des hommes, il sonde leur cœur ${ }^{68}$. Chrétien de Troyes, quant à lui, voyait dans le cœur le lieu où s'accomplit l'union mystique avec le plus pur de nous-mêmes ${ }^{69}$,

62. Mary J. Carruthers, The Book of Memory : A Study of Memory in Medieval Culture [1990], 2e éd., Cambridge, Cambridge University Press, 1994, p. 48.

63. Isidore de Séville, Etimologías, XI, I, 118-127, éd. Wallace Martin LindSAY, trad. José Oroz Reta et Manuel-A. Marcos Casquero, Madrid, BAC, 1983, t. II, pp. 32, 34.

64. Hildegarde de Bingen, Causae et curae, II, 1-12, éd. Paul Kaiser, Leipzig, Teubner, 1903, p. 43.

65. Id., Scivias, I, 4, 16, éd. A. Führkötten et A. Carlevaris, Turnhout, Brepols, 1978 (CCCM, 43), p. 78, 1. 577-578. Id., Causae et curae, II, 34-35, op. cit., p. 42.

66. Id., Scivias, III, 2, 21, op. cit. (CCCM, 43 A), p. 367, 1. 620.

67. Bernard de Clairvaux, In Nativitate Beatae Mariae, 3, éd. Jean Leclercq, trad. Mariano Ballano, Madrid, BAC, 1983 («Obras Completas de San Bernardo »), t. IV, p. 420. Id., De sancta Maria, 2, ibid., t. VI, p. 344.

68. Pierre Abélard, Ethics, éd. et trad. D. E. Luscombe, Oxford, Clarendon Press, 1971, p. 43.

69. Chrétien de Troyes, Cligès, vv. 708-716, op. cit., p. 22. 
puisque cet organe est le siège de l'amour ${ }^{70}$, de la mémoire ${ }^{71}$, de la $v^{7} \mathrm{e}^{72}$. Vincent de Beauvais considérait le cœur comme le principal « organe spirituel ${ }^{73} »$. L'évolution de la hiérarchie des sens n'affecta pas l'importance attribuée au cœur: si l'on parlait auparavant de «l'ouïe du cœur», avec les troubadours et les romans courtois on en vint à associer l'œil et le cœur ${ }^{74}$. Au début du XIII ${ }^{\mathrm{e}}$ siècle, un poème établit une relation entre le cœur et le phallus, le sentiment et la sexualité, en racontant l'histoire d'un personnage tué par les maris de ses maîtresses, lesquels lui arrachèrent ces deux organes et les donnèrent à manger à leurs épouses adultères ${ }^{75}$.

70. Ibid., vv. 4302-4306, p. 131.

71. Chrétien de Troyes, Le Conte du Graal ou le Roman de Perceval, v. 3235, éd. et trad. Charles Méla, Paris, LGF, 1990 (coll. « Le Livre de poche. Lettres gothiques »), p. 242. Depuis lors, le cœur est devenu le siège par excellence de la mémoire émotive pour la culture occidentale. Au XIII ${ }^{\mathrm{e}}$ siècle, parmi beaucoup d'autres exemples possibles, rappelons l'histoire des deux dévots dont on ouvrit le cœur après la mort: sur l'un était inscrit le nom de Jésus-Christ (Jacques de Voragine, Legenda aurea, XXXVI, op. cit., p. 157), tandis que l'autre contenait une image de la Vierge (Cantigas de Santa María, 188, op. cit., t. II, pp. 213-214) ; de même, les stigmates reçus par saint François d'Assise s'étaient " profondément imprimés dans son cœur » dès que le crucifix de l'église Saint-Damien lui avait parlé (Thomas de Celano, Vita seconda di San Francesco d'Assisi, I, VI, 10, trad. S. Colombarini, in Fonti francescane, $4^{\mathrm{e}}$ éd., Padoue, Messaggero, Movimento Francescano, 1980, p. 562).

72. Chrétien de Troyes, Cligès, vv. 3668-3673, op. cit., p. 112. Id., Erec et Enide, vv. 2869-2870, éd. Mario Roques, trad. René Louis, Paris, Honoré Champion, 1984 (Traductions des CFMA), p. 75.

73. Vincent de Beauvais, Speculum historiale, I, 32, Graz, Akademische Drunck-u. Verlagsanstalt, 1965, p. 13.

74. Guy Paoli, «La relation œil-cœur. Recherches sur la mystique amoureuse de Chrétien de Troyes dans Cligès », Senefiance, 30, 1991, pp. 233-244.

75. Lai d'Ignauré, trad. Danielle Régnier-Bohler, in Le Couur mangé : récits érotiques et courtois des XII et XIII siècles, Paris, Stock, 1979, pp. 221-239. Naturellement, comme l'a signalé Milad Doueihi (Histoire perverse du cour humain, trad. Pierre-Antoine Fabre, Paris, Éd. du Seuil, 1996, pp. 38-55), la scène du cœur dévoré est une parodie de la Cène (les amantes étaient au nombre de douze, et après avoir su ce qu'elles venaient de manger, elles ne s'alimentèrent plus jamais) et de l'eucharistie (par ce macabre repas, elles avaient rencontré l'amant désiré et absent, avaient fusionné avec lui, et finalement s'étaient réunies à lui dans la mort, cherchée par le refus d'autres aliments). On doit voir aussi Luciano Rossi, «Suggestion métaphorique et réalité historique dans la légende du coeur mangé », Micrologus, 11, 2003, pp. 469-500. 
Le nouveau sentiment collectif en relation avec le cœur est resté présent dans les idiomes alors en formation. Du latin classique cor, synonyme de « mémoire » (et aussi de « pensée », d' " intelligence », $\mathrm{d}^{\prime}$ « $\left.\mathrm{a} m \mathrm{e}^{76} »\right)$ sont sortis en français « recorder », en italien ricordari, en castillan et en portugais recordar. Si le cœur comme centre de l'acte mémoriel apparaît dans la racine des mots castillan et portugais decorar, il est encore plus explicite dans les locutions française " par cœur» (apparue aux alentours de 1200), portugaise de cor (datée du XIII ${ }^{\mathrm{e}}$ siècle) et anglaise by heart (attestée vers 1374, et basée sur l'acception de herte comme "mémoire », existant depuis le début du XII ${ }^{\mathrm{e}}$ siècle ${ }^{77}$ ). Toutefois, le cœur n'était pas considéré seulement comme le siège de la mémoire. En anglais, il était lié au courage (vers 825), aux émotions (1050), à l'amour (vers 1175), au caractère $(1225)^{78}$. Dans l'italien médiéval, le cœur (core avant 1250, puis cuore) était réputé être le centre des sentiments, des émotions et de la pensée ${ }^{79}$.

Le plus souvent, l'association s'est faite entre l'organe et un sentiment dont on pensait qu'il en provenait directement, comme l'attestent diverses langues occidentales : en français curage (apparu en 1080, écrit ensuite courage, utilisé comme synonyme de cœur jusqu'au XVII ${ }^{\mathrm{e}}$ siècle), en italien coraggio (antérieur à 1257), en castillan coraje et en portugais coragem (tout les deux du XIV siècle), en allemand herzhaftigleit (mot du XV siècle dérivé de herz, « cœur », écrit herza au VIII ${ }^{\mathrm{e}}$ siècle), en anglais courage (vers 1500, aux environs de 1300 écrit corage). Cette dernière langue présente un cas intéressant, qui montre l'hésitation psycho-culturelle entre le foie et le cœur comme siège des sentiments positifs : «lâchement» est désigné

76. Ce serait encore le sens du mot pour saint Augustin, selon Edgardo de la Peza, El Significado de «cor » en San Agustin, Paris, Études augustiniennes, 1962.

77. A. Rey, Dictionnaire historique..., op. cit., t. I, p. 442. José Pedro Machado, Dicionário etimológico da língua portuguesa, 6e éd., Lisbonne, Horizonte, 1990, t. II, p. 288. The Oxford English Dictionary, op. cit., t. V, p. 161.

78. The Oxford English Dictionary, op. cit., t. V, p 159.

79. Manlio Cortelazzo et Paolo Zolli, Dizionario etimologico della lingua italiana, Bologne, Zanichelli, 1980, t. I, p. 305. Selon le même processus, en roumain, le mot est inima, dérivé du latin anima. 
par le mot composé liver-heartedness, littéralement «sans foie ni cœur». Une autre preuve de l'importance morale attachée à cet organe se retrouve dans le mot cordial, qui à l'origine avait le sens neutre de « relatif au cœur», et qui plus tard allait acquérir celui, positif, de « gentil », « aimable », tant en français, en anglais, en castillan et en portugais, qu'en italien (cordiale) et en allemand (herzlich).

La valorisation symbolique du cœur survenue au XII siècle était aussi présente dans la culture juive. Alors que le Pirkei Rabbi Nathan, un texte antérieur au $\mathrm{X}^{\mathrm{e}}$ siècle, établit plusieurs comparaisons entre des parties de l'univers et des parties du corps humain et ne cite même pas le cour, Maïmonide, dans la deuxième moitié du XII ${ }^{\mathrm{e}}$ siècle, considère ce dernier comme le centre du corps humain ${ }^{80}$. Il s'inspire probablement ici d'Aristote, pour qui le corps humain se développe à partir du cœur, idée très influente à partir de la redécouverte chrétienne du Stagirite. Ainsi, certaines représentations romanes de la création d'Adam le montrent prenant vie non par un « souffle sur le visage » (in faciem eius spiraculum vitae) comme le dit le texte biblique ${ }^{81}$, mais par le toucher de la main de Dieu sur son cœur. C'est le cas, par exemple, d'un manuscrit de l'abbaye de Saint-Martial de Limoges ${ }^{82}$, enluminé aux environs de l'an 1100, et d'un relief sculpté quelques années après sur la façade nord de la cathédrale de Compostelle.

L'importance du cœur dans la culture romane transparait aussi dans son utilisation métaphorique grandissante. Sur le plan politique, il devint le « roi » du corps humain, de la même façon que le roi est le «cœur» du corps social ${ }^{83}$. Sur le plan littéraire se propagea la figure rhétorique du cœur comme un livre sur lequel soit un individu commun, soit un saint, soit même le Christ, inscrit ses émotions

80. Samuel S. Kottek, «Microcosm and Macrocosm according to some Jewish medieval works up to the 12th century », Janus, 64, 1977, pp. 206-211.

81. Genèse, II, 7.

82. Breviarium ad usum S. Martialis Lemovicensis, Paris, BNF, lat. 743, fol. $112 \mathrm{v}$.

83. Jacques Le Goff, « Head or Heart ? The political use of body methaphors in the Middle Ages », in Michel Feher, éd., Fragments for a History of the Human Body, New York, Zone, 1989, pp. 12-27. 
amoureuses (y compris érotiques) et spirituelles ${ }^{84}$. Sur le plan architectural, le schéma cruciforme des églises plaçait l'autel - lieu où se reproduit le mystère de l'incarnation - dans la position occupée par le cœur ${ }^{85}$. Sur le plan liturgique, la christianisation du Graal en fit le récipient contenant le sang du Christ, le transformant symboliquement en un cœur ${ }^{86}$. Sur le plan géographique, de la même façon que le cœur était le centre du corps humain, le sépulcre du Seigneur était le cœur du monde, selon un sermon de Pierre le Vénérable ${ }^{87}$. Sur le plan linguistique, dès le $\mathrm{XIII}^{\mathrm{e}}$ siècle, le mot désignait en français et en italien le centre de quelque chose, comme par la suite en anglais (début du XIV siècle) et en castillan (XVI ${ }^{\mathrm{e}}$ siècle) ${ }^{88}$. Quand, dans ce contexte culturel, l'abbesse de Bingen affirmait que l'Adam fait en argile était un corps vide avant d'avoir été rempli par Dieu avec un cœur, un foie, des poumons, un estomac et des viscères ${ }^{89}$,

84. Sur l'évolution de cette métaphore, Ernst Robert Curtius, La Littérature européenne et le Moyen Âge latin [1947], trad. Jean Bréjoux, Paris, PUF, 1986, t. II, p. 30 ; Eric Jager, «The Book of the Heart : reading and writing the medieval subject », Speculum, 71, 1996, pp. 1-26.

85. Ce n'est pas un hasard si en français médiéval le même nom désigne cette partie de l'église et l'organe : cuer, mot apparu en 1080 dans son sens anatomique et vers 1150 dans son sens architectural. Le changement culturel et, par conséquent, orthographique postérieur - chœur est attesté en 1568 - ne cache pas cette origine commune, puisque la langue a conservé l'homonymie des mots.

86. Begoña Aguiriano, «Le cour dans Chrétien », Senefiance, 30, 1991, p. 19. Le cœur apparaît plusieurs fois et avec des significations multiples dans le cycle du Lancelot-Graal : voir Micheline de Combarieu du Grès, « Un cœur gros comme ça (Le cœur dans le Lancelot-Graal) », ibid., pp. 77-105.

87. Pierre le Vénérable, In laudem sepulcri Domini, PL, t. CLXXXIX, col. 978 d-979 a. Pour la métaphore du coeur comme soleil du corps humain, Thomas Ricklin, « Le coeur, soleil du corps : une redécouverte symbolique du XII ${ }^{\mathrm{e}}$ siècle », Micrologus, 11, 2003, pp. 123-143.

88. Cette acception avait été appliquée à la ville par Aristote dans son De motu animalium (trad. Martha C. Nussbaum, Princeton, Princeton University Press, 1978, pp. 50-52), et fut utilisée à la fin du Moyen Âge selon Roberto Lambertini, « Il cuore e l'anima della città. Osservazioni a margine sull'uso di metafore organicistiche in testi politici bassomedievali », in Carla Casagrande et Silvana Vecchio, éd., Anima e corpo nella cultura medievale, Florence, Galluzzo, 1999, pp. 289-304.

89. Hildegarde de Bingen, Causae et curae, II, 20, op. cit., p. 42. 
elle établissait apparemment une hiérarchie des organes. Ainsi, l'importance grandissante que le Sacré Cœur de Jésus acquiert dans la spiritualité à partir du XII ${ }^{\mathrm{e}}$ siècle semble avoir été la conclusion d'un long processus de valorisation médicale et symbolique de cet organe ${ }^{90}$.

\section{L'HÉSITATION EXÉGÉTIQUE}

Un exemple intéressant de la concurrence entre la figue et la pomme dans la fonction symbolique de fruit défendu nous est fourni par les sculptures de la façade occidentale de la petite église rurale castillane de San Quirce, près de Burgos, terminée en 1147. Y sont figurés, en onze modillons, plusieurs épisodes du mythe adamique, depuis la création des protoplasmes jusqu'à la condamnation de Caïn, tandis que dans les intervalles, dix métopes montrent des scènes parfois difficiles à mettre en relation avec celles des modillons, bien que chaque étape du cycle soit identifiée par des inscriptions ${ }^{91}$. L'ensemble forme un discours iconographique à deux rythmes, dont le sujet est le mal, tant à son origine (péché originel) que dans quelques-unes de ses manifestations (sexe, mort, impureté corporelle).

Ce dernier aspect est visible sur les deux métopes des extrémités, où l'artiste a représenté un homme déféquant. Il ne s'agissait pas de simple curiosité ni d'obscénité, car la disposition de ces scènes est

90. Jean-Vincent Bainvel, « Cœur sacré de Jésus (dévotion au)», Dictionnaire de théologie catholique, op. cit., t. III, col. 305 et suiv. On peut se demander si la "nouvelle phase » de cette dévotion au XVII ${ }^{\mathrm{e}}$ siècle (ibid., col. 313-314), n'était pas la conséquence, sur le plan mystique, des découvertes de William Harvey sur la circulation sanguine et le cœur (De motu cordis et sanguinis in animalibus, 1628).

91. Ces inscriptions sont actuellement presque illisibles, mais elles ont été relevées par Justo Pérez de Urbel et Walter M. Whitehill JR., « La iglesia románica de San Quirce », Boletín de la Real Academia de la Historia, 98, 1931, pp. 801-803. 
significative, la première étant en mise en relation avec la faute d'Adam et la deuxième avec celle de Caïn. De fait, une inscription proche de la représentation du péché originel éclaircit l'articulation entre les événements montrés sur la métope et le modillon : MALA $C A G O$. Sans doute l'homme qui parle et agit de la sorte est-il à la fois l'Adam paradisiaque qui vient de manger les fruits défendus, et aussi le symbole de tous les êtres humains, ses «fils posthumes » comme le définissait un sermon contemporain ${ }^{92}$. Mais l'interprétation exacte de l'inscription pose un problème important.

Il y a quelques décennies, l'historiographie a vu là un jeu de mots, puisque cet individu évacue en même temps des " pommes » et des « $\operatorname{maux}^{93} »$. Cette interprétation se fonde sur trois éléments : l'inscription de la façade, la présence à l'intérieur de l'église d'un chapiteau sur le même sujet, qui montre indubitablement une pomme, enfin l'enracinement pluriséculaire de la tradition qui voit dans ce fruit l'aliment défendu du Paradis. Toutefois, sur la scène du modillon, les fruits défendus ressemblent plutôt à des figues, une impression que renforce une explication non formaliste. En effet, la figue possède traditionnellement un caractère explicitement sexuel, tandis que la pomme, bien que liée à la déesse de l'amour Aphrodite, avait une connotation plus sensuelle que précisément sexuelle. C'est ce que nous montre par exemple une saga islandaise du XIII ${ }^{\mathrm{e}}$ siècle, dans laquelle le philtre amoureux est une pomme, ou encore, dans certaines mythologies, les vertus rajeunissantes et embellissantes qui lui sont attribuées, et qui subsistent dans l'étymologie de " pommade », substance parfumée, cosmétique et guérissante à base de pomme ${ }^{94}$.

92. Julien de Vézelay, Sermons, XV, éd. et trad. Damien Vorreux, Paris, Éd. du Cerf, 1972 (coll. « Sources chrétiennes », 192), p. 304.

93. J. Pérez de Urbel et W. M. Whitehill, « La iglesia románica de San Quirce », art. cit., p. 801. Luis Maria de Lojendio, Castilla 1, Madrid, Encuentros, 1978 (« La España románica », 1), p. 285. Manuel Guerra Gómez, Simbología románica, Madrid, Publicaciones de la Fundación universitária española, 1978, p. 48.

94. Voir M. Pastoureau, «Bonum, malum, pomum... », art. cit., p. 170. A. ReY, Dictionnaire historique..., op. cit., s.v. 
C'est une telle association de la figue avec la sexualité que semble exprimer, au troisième quart du $\mathrm{XII}^{\mathrm{e}}$ siècle, le programme iconographique du portail de l'église de Barret, en Poitou. Nous avons là, de chaque côté, trois chapiteaux établissant entre eux une relation spatiale et symbolique très courante dans l'imaginaire roman. En observant l'ensemble à partir du chapiteau le plus proche de l'entrée, du côté gauche, le premier représente un péché originel dont le fruit est la figue, le second, un personnage dans une pose franchement obscène, le troisième, double, montre sur une face un aigle et sur l'autre un monstre dévorant un mouton. Symétriquement, du côté droit, le premier chapiteau représente des lions adossés, le suivant, deux colombes s'embrassant, le dernier, un centaure et une colombe. Le message nous semble clair : le péché (c'est-à-dire, la figue, le sexe), mène à des actes antinaturels, érotiques, et ainsi à la mort de l'âme, dévorée par le démon (le rapace et le monstre), tandis que ceux qui adhèrent au Christ (le lion) seront des innocents (les colombes) qui s'embrassent dans la paix et dans la pureté, apaisant le côté animal qui existe dans tout être humain (les centaures).

En effet, l'acception sexuelle de la figue était admise par la culture classique et n'a pas disparu avec sa christianisation. Le figuier fut tout au long des siècles associé à Dionysos, et au moins dans sa version romaine, Bacchus, l'image du dieu était toujours sculptée dans le bois de cet arbre et un panier de figues était l'objet le plus sacré de ses fêtes, les bacchanales. En tant que protecteur des vergers, et particulièrement du figuier, Dionysos se confondait avec son fils né d'Aphrodite, Priape. Dans les processions en hommage à ce dieu de la fécondité, doté d'un pénis démesuré, on exhibait un grand phallus sculpté dans le bois du figuier, dont les feuilles étaient elles aussi vues comme un symbole ithyphallique ${ }^{95}$. Cette notion d'exubérance sexuelle est également présente chez l'apologiste chrétien Clément d'Alexandrie (vers 150-vers 250) dans sa version d'un épisode du

95. J. Brosse, Mythologie des arbres, op. cit., pp. 290-291. Cette connotation sexuelle de la figue se maintient encore aujourd'hui en Afrique du Nord, où elle est un synonyme des testicules, voir É. Mozzani, Le Livre des superstitions, op. cit., p. 747. 
mythe de Dionysos ${ }^{96}$. De même, quoiqu'il appelle le foie iecur et non ficatum, Isidore de Séville fait implicitement le rapport en affirmant que dans cet organe « résident la volupté et la concupiscence ${ }^{97}$ ».

À tout cela, il faut ajouter la popularité d'un geste, « faire la figue », qui était associé à ce fruit par son nom et par sa forme. Cette association est constatée par l'idiome castillan, où les deux mots (higo/higa) apparurent au même moment, aux environs de $1140^{98}$. Ce geste a pris dans la tradition populaire de plusieurs sociétés, et même dans celle de l'Occident médiéval, « une évidente connotation sexuelle ${ }^{99}$, qui peut se référer soit à la représentation de l'organe sexuel féminin (sens prédominant), soit à son état d'excitation (dans ce cas, le bout du pouce entre l'index et le majeur imite un clitoris tuméfié), soit à une copulation (dans ce cas, le pouce est le pénis situé entre les lèvres vaginales), soit à un phallus (sens plus rare $)^{100}$. C'est probablement dans ce dernier sens qu'autrefois, en Bavière, le jeune homme qui prétendait se marier envoyait une figue

96. Clément d'Alexandrie, Protreptique, II, 34, 3-4, éd. et trad. Claude Mondésert et André Plassart, 2e éd., Paris, Éd. du Cerf, 1976 (coll. «Sources chrétiennes », 2bis), pp. 90-91 : «Dionysos désirait vivement descendre chez Hadès, mais il ignorait le chemin ; un certain Prosymnos promet de le lui expliquer, mais contre salaire, un salaire qui n' avait rien de beau, sinon pour Dionysos : on lui réclamait de se prêter aux plaisirs de l'amour. Le dieu accueille volontiers la demande, il promet d'y répondre s'il revient et confirme par serment sa promesse. Renseigné, il s'éloigne ; il revient et ne trouve pas Prosymnos (qui était mort); pour s'acquitter envers son amant, Dionysos se rend, plein de désirs impurs, à son tombeau. Il coupe, au hasard, une branche de figuier, lui donne la forme voulue et s'en sert pour remplir, à l'égard du mort, sa promesse. »

97. Isidore de Séville, Etimologías, XI, I, 125, op. cit., t. II, p. 34.

98. Joan Corominas, Diccionario critico etimológico de la lengua castellana, Madrid, Gredos, 1954, t. II, p. 916. En catalan, figa désigne aussi l'organe sexuel féminin, comme l'italien fica.

99. Jean-Claude Schmitt, La Raison des gestes dans l'Occident médiéval, Paris, Gallimard, 1990 («Bibliothèque des histoires »), p. 260.

100. D. Morris, P. Collet, P. Marsh et M. O’Shaughnessy, Os gestos : suas origens e significado [1979], trad., Lisbonne, Publ. Europa-América, s. d. [1984], pp. 191-195. Le sondage réalisé en Europe par ces auteurs entre 1975 et 1977 montre que sur 1200 personnes interrogées, 440 attribuaient encore une certaine connotation sexuelle à la figue, soit presque $37 \%$ du total. Si l'on ne considère que les personnes qui voyaient un sens à cette attribution, la proportion dépasse les $66 \%$ (ibid., p. 190). 
en or ou en argent à son amante, qui pouvait refuser la demande en rendant le cadeau, ou l'accepter en renvoyant un cœur en argent ${ }^{101}$.

Le far la fica était un geste agressif et péjoratif très utilisé par les Italiens du Moyen Âge, non seulement au quotidien, mais aussi dans des situations à forte charge émotionnelle. En 1162, irrité contre les Milanais qui avaient forcé sa femme à monter un mulet à l'envers, le devant tourné vers la queue de l'animal - une position très ancienne pour signifier le mépris - Frédéric Barberousse prit leur ville et, sous peine de mort, obligea les prisonniers à enlever avec les dents une figue placée dans l'anus d'un mulet ${ }^{102}$. Les habitants de Pistoia firent sculpter en leur château de Carmignano deux grands bras surmontés de mains faisant la figue vers la cité ennemie de Florence - laquelle, humiliée, alla conquérir la place en $1228{ }^{103}$. Chez Dante, un voleur condamné à l'Enfer fait la figue contre Dieu lui-même ${ }^{104}$. Le geste et l'expression ficha facere sont présents, avec le même sens dérisoire, dans l'ensemble des cultures romanes, et même en dehors de celles-ci ${ }^{105}$. Si ce geste a une fonction talismanique, celle d'écarter le mauvais œil et d'autres dangers, cela semble être dû exactement à sa connotation sexuelle, conjuratoire de la stérilité contraire à la vie ${ }^{106}$.

101. José Leite de Vasconcelos, A figa, Porto, Araújo e Sobrinho, 1925, pp. 81-82. Selon le même ethnographe, il était commun, dans le monde romain, de mettre en relation la figue et le phallus (ibid., p. 86).

102. Cité par J. Leite de Vasconcelos, A figa, op. cit., p. 80 ; par Jerome Fried et Mary Leach, dir., Standard Dictionnary of Folklore, Mythology and Legend, New York, Funk and Wargall, 1950, t. I, p. 378 ; par D. Morris et al., Os gestos, op. cit., p. 197.

103. Giovanni Villani, Cronica, VI, 5, éd. Ignazio Moutier, Florence, Sansone Coen, 1844, t. I, p. 228.

104. Dante Alighieri, Divina Commedia, Inferno, XXV, 1-3, éd. Giuseppe Vandelli, 21e éd., Milan, Hoepli, 1979, p. 202. Cette connotation blasphématoire est la raison pour laquelle en 1553 un juif converti fut dénoncé à l'Inquisition pour avoir «fait des figues au Saint Sacrement », voir José Leite de Vasconcelos, A figa, op. cit., p. 48.

105. J. Leite de Vasconcelos, A figa, op. cit., pp. 42-56, 72, 76-81 et 90. Même constatation pour la Suisse alémanique (ibid., pp. 77-78), la Grèce (p. 81), la Bohême et la Norvège (p. 82).

106. Ibid., pp. 27-41, 57-59 et 91-93. 
En ce sens, la scène de la faute primordiale sur le troisième modillon de San Quirce, en plus d'adopter l'ancienne interprétation du péché originel comme étant un péché sexuel ${ }^{107}$, préparerait l'observateur à rencontrer, trois métopes plus loin, juste après l'expulsion du Paradis, une représentation de la relation charnelle entre les protoplasmes ${ }^{108}$. Ainsi, selon notre hypothèse, le mot malum n' aurait pas été ici utilisé dans son sens spécifique de " pomme », mais dans celui, plus ample, de « fruit à pulpe » (opposé à nux, « fruit à peau dure ») ${ }^{109}$,

107. Voir Martin ElzE, Tatian und seine Theologie, Göttingen, Vandenhoeck und Ruprecht, 1960 (Forschungen zur Kirchen und Dogmengeschichte, 9). Henry Chadwick, "Enkrateia », in Reallexikon für Antike und Christentum, Stuttgart, Hiersemann, 1960, t. V, col. 343-365. Raniero Cantalemassa, éd., Etica sessuale e matrimonio nel cristianismo delle origini, Milan, Vita e Pensiero, 1976 ( «Studia Patristica Mediolanensia », 5). Elaine H. Pagels, Adam, Ève et le serpent [1988], trad. Michel Miech Chatenay, Paris, Flammarion, 1989, pp. 35-72.

108. J. Pérez de Urbel et W. M. Whitehill («La iglesia románica de San Quirce », art. cit., p. 801) croient que le modillon représente le reproche divin fait à Adam et à Ève, et que la métope sur le sexe montre en réalité la scène du péché sans la présence du serpent. Comme une telle lecture du cycle de San Quirce implique une subversion de l'ordre chronologique des faits bibliques, ces deux érudits ont soutenu, de façon peu convaincante, qu'un « maçon ignorant y a mis en place les fragments du récit dans le plus grand désordre ».

109. Bien que la première acception ait fini par s'imposer dans le latin médiéval, elle n'a pas éliminé la deuxième, comme on l'observe dans l'usage de malum suivi d'un qualificatif. Tel est le cas, par exemple, de malum armeniacum pour l'abricot. Ou encore de malum granatum, appelé aussi malum romana, pour le fruit qui tire son nom français de la première formule (grenade), et son nom portugais de la seconde (romã). C'est encore le cas de malum terrae pour les diverses racines comestibles (comme la mandragore) ou pour les végétaux ras (comme le potiron). Traduite en français au milieu du XVII ${ }^{\mathrm{e}}$ siècle, l'expression « pomme de terre » a fini par être employée pour désigner le tubercule américain dont l'usage se répandait alors, et qui a fini par s'imposer sur le mot antérieur («patate», de la fin du XVI ${ }^{\mathrm{e}}$ siècle). De façon générale, dans les parlers romans, le terme indéfini a disparu : par exemple, malum persicum a donné le français «pêche », l'italien pesca, le castillan prisco (qui a perdu sa place au profit de melocotón, dérivé de malum cotoniu), le portugais pêssego, le roumain piersica, le provençal persega, le catalan pressec. Dans ce cas, le terme indiquant l'origine géographique a prévalu, de la même façon que dans les langues germaniques « pomme » dérive du nom de la ville d'Abella, près de Naples, réputée dans l'Antiquité pour la production de ce fruit : aphul en bas allemand, apfel en allemand moderne, apple en anglais, appel en hollandais, eple en norvégien, aeble en danois, äple en suedois, epli en islandais. 
de sorte que le jeu de mots de l'inscription signifierait «évacuer des maux et des fruits ». Conscient ou non de l'ambiguïté de l'inscription, le sculpteur de San Quirce a ainsi révélé l'intéressante cohabitation des deux traditions exégétiques, celle de la pomme, présente dans la figuration du péché originel à l'intérieur de l'église, et celle de la figue, visible sur sa façade. Cohabitation plus expressive encore si l'on admet qu'un seul artiste a sculpté le chapiteau et le modillon ${ }^{110}$.

Cette hésitation exégétique n'a rien d'un cas isolé, survenu dans une communauté monastique du centre de la Castille. La formation du mot français « pomme » donne une intéressante indication dans ce sens. Même si, dès le début du ve siècle, le mot latin pomum (« fruit », au sens générique) a reçu le sens spécifique de «fruit du pommier » en Italie du Nord et dans la majeure partie du territoire ibéro-romain - sens conservé par le provençal et le catalan pomal'italien, le castillan, le portugais et le galicien ont fini par privilégier la forme classique malum, de laquelle ils ont tiré mela, manzana, maçã et mazál11. Pomum a conservé son acception large dans ces quatre langues, sous la forme pomo (poma dans le cas du galicien). Par la même évolution, c'est du latin classique pomarium que sont sortis les collectifs pomario en italien et pomar en castillan, portugais, provençal et galicien.

Différemment, le latin médiéval de la Gaule disposait, depuis la fin du viII siècle, du mot pomarius pour indiquer le pommier, d'où

110. Fait que L. M. de Lojendio (Castilla 1, op. cit.) considère comme possible, alors que J. Pérez de Urbel et W. M. Whitehill («La iglesia románica de San Quirce », art. cit., p. 812) pensent qu'il s'agissait d'artistes différents. De toute façon, les trois auteurs croient que le chapiteau et le modillon ont été sculptés dans un intervalle court, confirmant ainsi la coexistence des deux exégèses.

111. Tant le mot espagnol manzana (attesté en 1112 comme mazana) que le portugais maçã (attesté comme toponyme en 1187: Maçãas) dérivent de mala mattiana, fruit de la région de Mattium (l'actuelle Marbourg), voir J. Corominas, Diccionario critico etimológico..., op. cit., t. III, p. 248. J. P. Machado, Dicionário etimológico..., op. cit., t. II, p. 1450. Antenor Nascentes, Dicionário etimológico da lingua portuguesa, Rio de Janeiro, Francisco Alves, 1932, p. 307. Le roumain a connu une évolution semblable, avec $\operatorname{mar}$ (« pomme ») et poama (« fruit »). 
dériva en 1080 le nom vernaculaire de ce fruit spécifique (pume) à partir du terme générique (pomum) ${ }^{112}$. À la même date apparaissait, pour désigner le terrain planté de différents arbres fruitiers, le français verger, tiré du latin viridiarum (de viridis, «vert»). Devant ces données, il n'est pas absurde de présumer que l'évolution linguistique française refusait inconsciemment le caractère négatif supposé de ce fruit, caractère présent dans le mot malum, alors que la pomme est un symbole positif pour la culture celte ${ }^{113}$, fortement présente dans le territoire de la future France, surtout dans le contexte de la « réaction folklorique » du XII ${ }^{\mathrm{e}}$ siècle ${ }^{114}$.

C'est en fonction de son caractère archétypique, de fruit par excellence, que le mot a pu entrer dans la formation de tant de syntagmes, et même, vers 1256, dans l'expression curieuse " pomme de paradis », désignant la banane ${ }^{115}$. Mais si l'on constate, sur le plan du vocabulaire, une résistance française à l'association entre la pomme et le fruit du péché, sur le plan iconographique une telle identification s'établit, on l'a vu, sans problème. Il en fut de même pour les œuvres littéraires de portée populaire, tel le premier texte théâtral français, du milieu du XII ${ }^{\mathrm{e}}$ siècle, ou un sermon de la même

112. Le mot apparaît dans la Chanson de Roland comme pume ; en 1155, il est devenu pome; en 1273, il a pris sa forme moderne de pomme, voir A. Rey, Dictionnaire historique..., op. cit., s.v. L'hésitation vis-à-vis du mot employé est révélée par l'existence de l'expression pommes maciennes: Frédéric Godefroy, Dictionnaire de l'ancienne langue française et tous ses dialectes $d u I X^{\mathrm{e}}$ au $X V^{\mathrm{e}}$ siècle, Paris, Librairie des Sciences et des Arts, 1938, t. V, p. 60 ; Antoine Thomas, «Mots obscurs et rares de l'ancienne langue française », Romania, 36, 1907, pp. 278 et 280.

113. Françoise Le Roux et Christian-Joseph Guyonvarc'h, Les Druides, Rennes, Ouest-France, 1986, p. 413 ; l'au-delà celte, Avalon, signifie « île des pommes » (ibid., p. 466).

114. Jacques Le Goff, «Culture cléricale et traditions folkloriques dans la civilisation mérovingienne » [1967], in id., Pour un autre Moyen Âge, Paris, Gallimard, 1977 (« Bibliothèque des histoires »), p. 233, n. 26.

115. A. Rey, Dictionnaire historique..., loc. cit. Il est intéressant de remarquer qu' au XVII ${ }^{\mathrm{e}}$ siècle, un hérétique portugais qui avait habité plusieurs années au Brésil considérait la banane courte comme le fruit défendu: Processo da Inquisição contra Pedro Rates Hanequin, II, 7, édité et cité par Plinio Freire Gomes, Um herege vai ao Paraíso, São Paulo, Companhia das Letras, 1997, p. 166. 
époque $^{116}$. C'est aussi le cas, au même siècle et au siècle suivant, de diverses histoires d'amour commençant généralement par une trahison (des cœurs métaphoriquement dévorés) et finissant avec la mort des deux protagonistes (l'un d'eux dévorant littéralement, sans le savoir, le cœur de l'autre $\left.{ }^{117}\right)$. Dans une certaine mesure, ces récits réécrivaient, consciemment ou inconsciemment, le drame de la chute originelle : trahissant la confiance du Créateur (« de l'arbre... tu ne mangeras pas ») pour avoir mangé la pomme/cœur (« connaissance du bien et du mal »), l'être humain a provoqué sa propre perdition (« le jour où tu en mangeras, tu deviendras passible de mort ») car Adam comme Ève avaient les cours pleins de superbe (« vous serez comme des dieux $\left.{ }^{118} »\right)$.

\section{L'ARBRE ET L'ANDROGYNIE}

Cette quête de l'identité du fruit défendu roman doit encore considérer l'arbre par rapport au couple primordial. La position de ces trois éléments fournit d'importantes informations. Une des solutions symbolico-plastiques adoptées fut de représenter les primi parentes du même côté de l'arbre, Ève étant toujours plus proche de celui-ci (figure 4). La composition la plus répandue plaçait l'arbre entre Adam et Ève, comme l'avait déjà fait, dès la fin du III ${ }^{\mathrm{e}}$ siècle ou le début du IVe siècle, le sarcophage de San Justo de la Vega, à Léon, actuellement au musée archéologique de Madrid. Il serait réducteur de penser que cette position de part et d'autre de l'arbre, répondait simplement au désir de symétrie de l'art roman ${ }^{119}$,

116. Respectivement Le Mystère Adam: Ordo representationis Ade, vv. 109, 192, 305, 462 et 576, éd. Paul Aebischer, Genève, Droz, 1964, pp. 35, 43, 54, 62, 63 et 69, et Grant mal fist Adam, s. 13, éd. H. Suchier, in Reimpredigt, Halle, Max Niemeyer, 1879, p. 8. Ce texte avait initialement (s. 1., v. 3, p. 2) utilisé le terme générique et biblique « fruit».

117. Récits réunis in D. Régnier-Bohler, éd., Le Cour mangé, op. cit., et analysés par M. Doueihi, Histoire perverse du cœur humain, op. cit. supra, n. 74.

118. Genèse, II, 17 ; III, 5. Sur l'étroite relation entre savoir et saveur, Pierre Guiraud, Sémiologie de la sexualité, Paris, Payot, 1978, pp. 141-164.

119. Comme le croit M. Guerra, Simbología románica, p. 107. 


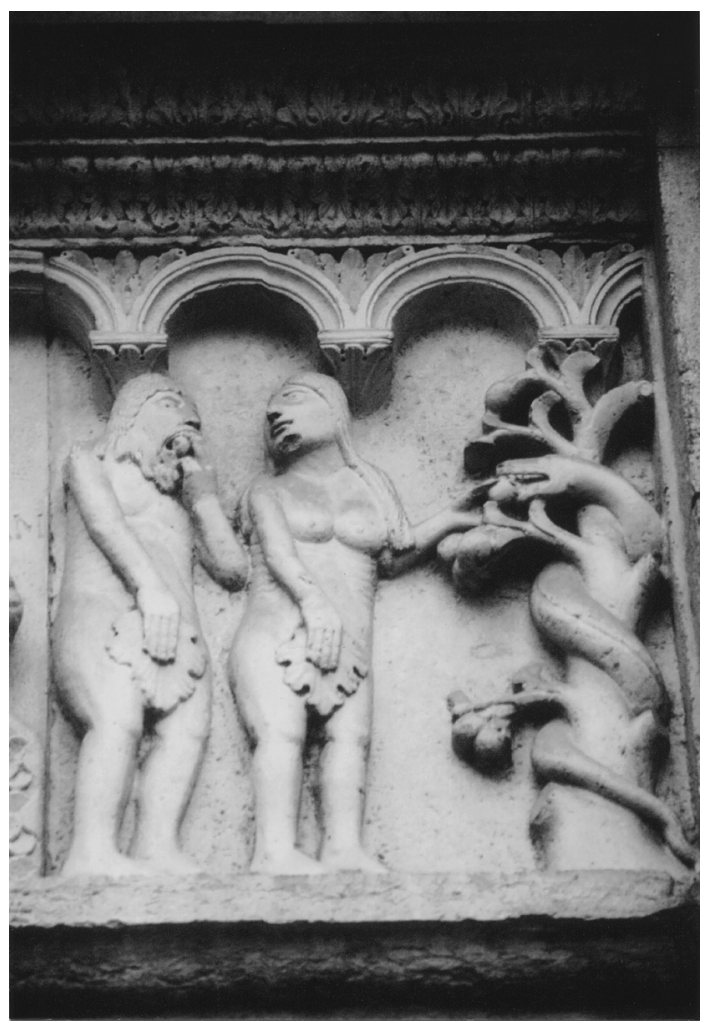

Figure 4. Relief de la façade occidentale de la cathédrale de Modène (Émilie-Romagne), vers 1100.

Photo de l'auteur

car la forme est presque toujours un fragment du contenu qui a émergé ${ }^{120}$. Aux $\mathrm{XI}^{\mathrm{e}}-\mathrm{XIII}{ }^{\mathrm{e}}$ siècles, ce schéma faisait probablement allusion à deux questions fortement présentes, liées au phénomène contemporain de sacralisation du mariage.

D'une part, en plaçant Adam et Ève à égale distance de l'arbre, l'iconographie faisait ainsi référence à un certain égalitarisme social et à un certain nivellement moral entre l'homme et la femme, même

120. Gerardus Van Der Leeuw, La Religion dans son essence et ses manifestations : phénoménologie de la religion, trad. Jacques Marty, nouv. éd., Paris, Payot, 1970, p. 451. 
si le serpent est presque toujours tourné vers la femme. Le côté où chaque personnage était placé variait. On a déjà considéré comme une «tradition iconographique » la position d'Ève à droite de l'arbre, schéma qui aurait à peine trois exceptions, à Saint-Antonin, à Bruniquel et à Lescure ${ }^{121}$. En fait, la femme apparait à gauche dans plusieurs autres cas, à titre d'exemple sur les sculptures d'Anzyle-Duc, Airvault, Butrera, Cergy, Cervatos, Covet, Embrun, Gémil, Gérone, Lavaudieu, Lescar, Loarre, Luc-de-Béarn, Mahamud, Manresa, Moirax, Montcaret, Peralada (figure 6), Saint-Étienne-de-Grès, Saint-Gaudens, Sangüesa, San Juan de la Peña, Toirac, Vérone et Vézelay. De même, sur les fresques d'Aimé, Fossa et San Justo de Ségovie, sur les enluminures de la Bible de Burgos, de l'Exultet 3 de Troia et de l'Hortus Deliciarum, sur un médaillon métallique de Saint-Jean-de-Latran, et sur les mosaïques de Monreale et de Trani.

D'autre part, la position centrale de l'arbre, séparant Adam et Ève, insinuait une rupture de l'unité initiale, existante au moins sur le plan psychologique. L'arbre, c'est-à-dire la connaissance, révélait la présence de traits contradictoires chez l'être humain, fait à l'image et à la ressemblance de Dieu, l'androgyne par excellence. «Dieu créa l'homme à son image, à l'image de Dieu il le créa, homme et femme il les créa ${ }^{122} »$ : c'est pourquoi l'être humain était initialement double, et donc par essence complet, microcosmique ${ }^{123}$. Retirer Ève de la côte d'Adam avait été une chirurgie de séparation, car ils étaient formés des mêmes os, ils étaient « une seule chair ${ }^{124}$ ». C'est ainsi que le texte sacré fut interprété dès la première moitié du premier siècle par le juif Philon d'Alexandrie, et à sa suite par Ambroise, Augustin, Grégoire le Grand, Isidore, le pseudo-Rémi d'Auxerre,

121. Jean-Claude Fau, « Découverte à Saint-Antonin (Tarn-et-Garonne) d'un chapiteau roman consacré à Adam et Ève », Bulletin monumental, 135, 1977 , pp. 233 et 235.

122. Genèse, I, 27.

123. Il y avait plusieurs types d'homme microcosmique dans la pensée de l'époque romane, comme l'a démontré Ruth Finckh, Minor Mundus Homo : Studien zur Mikrokosmos-Idee in der mittelalterlichen Literatur, Göttingen, Vandenhoeck und Ruprecht, 1999, pp. 116-320. Pour l'aspect iconographique, voir Fritz Saxl, «Macrocosm and Microcosm in Medieval Pictures », in id., Lectures, Londres, Warburg Institute, University of London, 1957, t. I, pp. 58-72.

124. Genèse, II, 23-24. 
Guibert de Nogent, Pierre Lombard, Bernard et d'autres, tous voyant Ève comme l'image de la femme intérieure de l'homme ${ }^{125}$.

Augustin, notamment, reconnaissait implicitement l'androgynie du premier homme lorsqu'il disait que le diable « ne peut pas nous tenter que par le biais de cette partie animale qui se révèle en un homme unique comme une image ou un exemplaire de la femme ${ }^{126} »$. Suivant un raisonnement d'essence paulinienne, il voyait en AdamÈve la complémentarité esprit-chair, comparaison qui fut adoptée à l'époque romane par beaucoup de penseurs. Comme, dans le texte biblique, «Adam » est à l'origine le nom générique attribué à l'être humain (Genèse, I, 19) et devient nom de personne seulement par la suite (Genèse, III,17), Augustin interprétait le mot « homme » (Genèse, I, 26) comme la « nature humaine ${ }^{127}$ ». Saint Anselme, très influent au XII ${ }^{\mathrm{e}}$ siècle, était d'accord pour considérer que, par «Adam », il fallait initialement comprendre Adam et Ève ${ }^{128}$. En essayant d'expliquer comment l'interdiction du fruit faite à Adam impliquait aussi Ève, Pierre Comestor affirmait qu'à travers l'homme elle fut transmise à la femme ${ }^{129}$; il sous-tendait ainsi implicitement l'unicité des deux personnages, l'androgynie de l'être auquel il fut interdit de manger le fruit.

Bien que l'Église médiévale n'ait pas formellement accepté l'androgynie divine et adamique, celle-ci ne lui était pas inconnue. Elle était présente dans un texte néo-testamentaire : «Il n'y a ni homme ni femme, puisque vous tous êtes un seul dans le Christ ${ }^{130}$. » Elle apparaissait dans un texte apocryphe : «Lorsque vous ferez le

125. Michel Planque, «Ève», in Dictionnaire de spiritualité, Paris, Gabriel Beauchesne, 1961, t. IV-2, col. 1773-1778.

126. Augustin, Del Genesis contra los maniqueos [De Genesi contra manichaeos], II, 18, 28, éd. et trad. Balbino Martin, $2^{\mathrm{e}}$ éd., Madrid, BAC, 1969 («Obras de San Agustin », 15), p. 378. Voir aussi id., Enarratio in Psalmos, XLVIII, I, 6, PL, t. XXXVI, col. 548.

127. Augustin, De Trinitate, I, 7, PL, t. XLII, col. 829.

128. Anselme de Cantorbéry, La Conception virginale et le Péché originel [De conceptu virginali et de originali peccato], IX, éd. et trad. Michel Corbin et Alain Lauras, Paris, Éd. du Cerf ( «Euvres de saint Anselme de Cantorbéry », 4), 1990, pp. 156-158.

129. Pierre Comestor, Historia scholastica, 15, PL, t. CXCVIII, col. 1069 a.

130. Galates, III, 28. 
deux Un et que vous ferez l'intérieur comme l'extérieur, l'extérieur comme l'intérieur, le haut comme le bas, lorsque vous ferez du masculin et du féminin un Unique, afin que le masculin ne soit pas un mâle et que le féminin ne soit pas une femelle [...] alors vous entrerez dans le Royaume [de Dieu ${ }^{131}$ ]. » Elle constituait une part non méprisable de la pensée de Clément d'Alexandrie ${ }^{132}$ (vers 150vers 215), d'Origène ${ }^{133}$ (185-254), de Grégoire de Nysse ${ }^{134}$ (vers 330390) et, à travers eux, de Jean Scot Érigène ${ }^{135}$ (vers 810-870). Elle avait sans doute fait partie de l'ambiance culturelle et psychologique des premiers siècles chrétiens ${ }^{136}$.

Or, si l'androgyne édénique avait disparu, ce devait être à cause du péché. Pour quelques penseurs, c'est ainsi que l'être humain avait pris conscience de sa duplicité, depuis lors rompue et marquée par le sexe, preuve visible de la faute originelle : sexus vient de sectio (« coupure», « séparation »), terme dérivé de secare, « couper », qui seulement au Moyen Âge reçut une acception spécifiquement sexuelle ${ }^{137}$. Il

131. Il Vangelo di Tommaso, 22, trad. Mario Erbetta, Casale Monferrato, Marietti, 1975, t. I, p. 267, 1. 17-23 et 26-27.

132. Dans une œuvre aujourd'hui perdue, Hypotyposes, selon Pierre Batiffol, Ancienne littérature chrétienne, Paris, Lecoffre, 1906, p. 162.

133. Selon lui, se fondant sur Luc, $x x, 36$, il n'y aura pas des sexes après la résurrection : Origène, Contre Celse [Contra Celsum], IV, 29, éd. et trad. Marcel Borret, Paris, Éd. du Cerf, 1968 (coll. « Sources chrétiennes », 136), p. 253.

134. Grégoire de Nysse, La Création de l'homme [De opificio hominis], 177d-192a, éd. et trad. Jean Laplace et Jean Daniélou, Paris, Éd. du Cerf, 1943 (coll. « Sources chrétiennes », 6), pp. 151-166 ; ibid., 204b-205c, pp. 183-186.

135. Jean Scot Érigène, Periphyseon, IV, PL, t. CXXII, col. 793 b-799 c. Pour des commentaires importants sur le sujet, Édouard Jeauneau, «La division des sexes chez Grégoire de Nysse et chez Jean Scot Érigène », in id., Études érigéniennes, Paris, Études augustiniennes, 1987, pp. 343-364.

136. Wayne A. Meeks, « The Image of the Androgyne : Some Uses of a Symbol in Earliest Christianity », History of Religions, 13, 1974, pp. 165-208.

137. Du Cange, Glossarium mediae et infimae latinitatis [1678], éd. L. Favre, rééd., Paris, Librairie des Sciences et des Arts, 1938, s.v. En latin classique, le groupe verbal secare n'avait aucune signification sexuelle, selon A. Ernout et A. Meillet, Dictionnaire étymologique..., op. cit., p. 622. On disait castrare pour l'amputation des organes génitaux (ou encore, quoique non spécifiquement, subducere, exsecare ou excidere), mais dans le latin chrétien, sectus, participe passé de secare, a pris le sens d' " eunuque », " châtré », voir Albert BLAISE, Dictionnaire latin-français des auteurs chrétiens, Strasbourg, Le Latin chrétien, 1954, p. 747. 
n'est donc pas aléatoire qu'Adam ait dit «moi » pour la première fois après le péché ${ }^{138}$. Si indéniablement le péché originel et le sexe étaient intimement liés, on discutait de la façon dont les choses s'étaient passées ${ }^{139}$. Un courant interprétait la faute comme un délit sexuel, par exemple le juif Philon et quelques Pères de l'Église, dont Clément d'Alexandrie et saint Ambroise ${ }^{140}$. À l'époque romane, la majorité des théologiens de l'école de Guillaume de Champeaux (1070-1121) pensaient également que ce péché avait été de concupiscence, quoique Guillaume lui-même le considérât comme une désobéissance à partir de laquelle la sensualitas parvint à dominer la ratio $^{141}$.

Un autre courant inversa la question et vit le sexe comme une conséquence du péché. Le Physiologus, influent traité allégoricozoologique traduit en latin au $\mathrm{V}^{\mathrm{e}}$ siècle, affirmait que l'éléphant et sa compagne, qui «personnifiaient» Adam et Ève, ignoraient le coït jusqu'au moment où la femelle avait mangé le fruit de la mandragore et l'avait donné au mâle : « à cause de cela ils durent sortir du Paradis ${ }^{142} \gg$. Le principal représentant de ce courant de pensée fut

138. «Mulier, quam dedisti mihi sociam, dedit mihi de ligno, et comedi »: Genèse, III, 12.

139. Emmanuele Testa, Il peccato di Adamo nella Patristica (Gen. III), Jérusalem, Tipographia dei PP. Francescani, 1970 (« Studii Biblici Franciscani Analecta », 3).

140. Philon d'Alexandrie, De opificio mundi, 151-152, trad. Roger Arnaldez, Paris, Éd. du Cerf, 1961 (« Euvres de Philon d'Alexandrie », 1), p. 243. Clément d'Alexandrie, Protreptique, XI, 1, op. cit., p. 179. Id., Stromata, III, 17, PG, t. VIII, col. 1206 b. Ambroise de Milan, Epistolae, LXII, 14, PL, t. XVI, col. 1193 a-b.

141. Odon Lottin, "Les théories du péché originel au $\mathrm{XII}^{\mathrm{e}}$ siècle", Recherches de théologie ancienne et médiévale, 11, 1939, pp. 20-21.

142. El Fisiólogo : bestiário medieval, 20, éd. Francis J. Carmody, trad. Marino Ayerra Redín et Nilda Guglielmi, Buenos Aires, Eudeba, 1971, pp. 6162. La mandragore était considérée un aphrodisiaque par le texte biblique (Genèse, XIV, 14-17), et ce caractère présumé était renforcé dans la culture médiévale par sa forme, qui rappelle en quelque sorte le corps humain : au $\mathrm{XII}^{\mathrm{e}}$ siècle, Hildegarde de Bingen observait qu'elle «est née de la terre avec laquelle Adam a été créé », et que, « à cause de sa ressemblance avec l'homme, la présence et les ruses du diable se font plus sentir en elle que dans les autres [plantes] » (Le Livre des subtilités des créatures divines (Physique), 56, trad. Pierre Monat, $3^{e}$ éd., Grenoble, Jérôme Millon, 1996, p. 72). 
saint Augustin, pour qui l'être humain d'avant le péché pratiquait le sexe sans concupiscence ${ }^{143}$. La faute du premier couple aurait alors été celle de l'orgueil, qui mena à la faute de la désobéissance, et de celle-ci à la faute charnelle ${ }^{144}$. Un autre défenseur de cette idée fut, au $\mathrm{VIII}^{\mathrm{e}}$ siècle, Jean Scot Érigène, qui pensait qu'avant le péché, l'être humain n'était qu'un, et que la division des sexes qui en découla cesserait dans la vie éternelle ${ }^{145}$. Sa pensée ne laissa pas d'exercer une certaine influence ; elle conduisit Maître Eckhart, au XIV ${ }^{\mathrm{e}}$ siècle, à considérer «toute division » comme "mauvaise en tant que telle » et voyait dans le chiffre deux le signe de la chute ${ }^{146}$.

Les représentations romanes de la faute initiale hésitaient dans le choix de l'une de ces positions théologiques. Indiquant la préférence pour la deuxième, plusieurs images accordèrent des attributs

143. Augustin, La Genèse au sens littéral [De Genesi ad litteram], IX, 6, op. cit., t. II, pp. 96-97 ; IX, 11, ibid., pp. 102-103 ; IX, 16, ibid., pp. 110-111. Id., La Cité de Dieu [De civitate Dei], XIV, 26, éd. Bernhard Dombart et Alfons Kalb, trad. Gustave Combès, Paris, Desclée de Brouwer, 1959 (« Euvres de saint Augustin », 35), pp. 458-459. Thomas d'Aquin, Somme théologique, I, q.98, a.2, trad. Marie-Jean Nicolas et al., Paris, Éd. du Cerf, 1984, t. I, p. 827. La tradition juive acceptait aussi l'idée d'activité sexuelle dans l'Éden (Midrach Rabba, XIX, 3, trad. B. Maruani et A. Cohen-Arazi, op. cit., p. 215 ; Génesis Rabbah I, trad. L. Vegas Montaner, op. cit., pp. 222-223), mais rien n'est dit à propos de son caractère moral.

144. Dans la première partie de son interprétation, Augustin se réfère conjointement à Genèse, III, où le péché est celui de l'orgueil, et à Romains, v, 19 , où il s'agit d'un péché de désobéissance (De Genesi ad litteram in duodecim, XI, 14-15, op. cit., t. II, pp. 256-263), mais quelques années plus tard, il considère que le sexe a été la conséquence et non la cause du péché : "nam corruptio corporis, quae adgravat animam, non peccati primi est causa, sed poena; nec caro corruptibilis animam peccatricem, sed anima peccatrix fecit esse corruptibilem carnem » (La Cité de Dieu, XIV, III, 2, op. cit., p. 358).

145. Jean Scot Érigène, Periphyseon, V, 20, PL, t. CXXII, col. 896 b.

146. Maître Eckhart, Commentaire de la Genèse, 88 et 90, op. cit., pp. 346 et 352. En effet, l'idée de l'unité comme perfection (par exemple, Bernard de Clairvaux, De consideratione, II, 8, 15, in "Obras Completas de San Bernardo », op. cit., t. II, p. 104) et donc de la division comme mal (étudiée par Gerschom Scholem, Von der mystischen Gestalt der Gottheit : Studien zu Grundbegriffen der Kabbala, Frankfurt am Main, Suhrkamp, 1962) nous semble être une réalité anthropologique plutôt qu'un élément de la culture judéo-chrétienne. Ainsi, les Timbiras, groupe indien de l'intérieur du Brésil, ne conçoivent pas l'opération mathématique $1+1$, puisque pour eux hommefemme, soleil-lune, feu-eau, etc., sont des ensembles, des globalités. 
sexuels à Adam et à Ève juste après l'ingestion du fruit : pour le premier, généralement la barbe ${ }^{147}$ (figures $1,2,3,4,5$ ), rarement un pénis (figure 5), pour la seconde, presque toujours des seins (figures 1, 2, 3, 4, 5, 6). D'autres images, minoritaires, semblent avoir vu dans la faute primordiale un acte sexuel, schéma iconographicothéologique manifesté peut-être pour la première fois, entre 1011 et 1015, sur la porte de bronze de la cathédrale allemande d'Hildesheim ${ }^{148}$. Adam y apparait à gauche de l'arbre, avec derrière lui un autre arbre sur lequel se tient un petit dragon. Ève est à droite, ayant près d'elle un autre arbre avec le serpent. Le fruit est la pomme, l'une dans la main droite d'Adam, l'autre dans la main droite d'Ève, tendue vers Adam. Il y a une autre pomme dans la main gauche de la femme, dont le bras plié se confond avec son vagin. Un schéma semblable fut utilisé à Rebolledo de la Torre, en 1186.

Dans la Bible d'Alard, le serpent qui donne le fruit à Ève est à la hauteur de son vagin, rappelant un membre sexuel masculin sur le point de réaliser une pénétration. La façade méridionale de SainteMarie de Sangüesa, en Navarre, de la deuxième moitié du XII siècle, semble exprimer la même conception. Là, la scène du péché se trouve immédiatement au-dessous de la personnification de la Luxure, montrant une femme dont les seins nus sont attaqués par des crapauds et des serpents ${ }^{149}$. Cette association luxure-péché originel n'était pas rare, et comme Sangüesa se trouvait sur la route compostellane de Jaca, la plus employée par les Occitans et les Italiens, nous pouvons penser à l'hypothèse que son message

147. Pour Hildegarde de Bingen, Causae et curae, II, 5-7, op. cit., p. 90, l'homme chevelu qui n'a pas une grande barbe est frigide et infertile, de faible masculinité.

148. William Tronzo, «The Hildesheim Doors : An Iconographic Source and Its Implications », Zeitschrift für Kunstgeschichte, 46, 1983, pp. 357-366.

149. Malgré la grande diversité du matériel iconographique de ce portail, qui fait penser au remploi tardif de pièces provenant d'autres parties de l'église, Luis Maria de Lojendio considère que cette œuvre possède une «admirable unité » (Navarra, Madrid, Encuentros, 1978 [«La España románica », 7], p. 167). Sur la formation de la représentation médiévale de la luxure, voir Jacqueline Leclercq-Kadaner, "De la Terre-Mère à la Luxure », Cahiers de civilisation médiévale, 18, 1975, pp. 37-43. 
iconographique manifestait le point de vue de beaucoup de pèlerins sur le sujet. Dans ce sens, l'image navarraise ratifiait au moins deux autres images connues de ces pèlerins.

La première, provençale, du deuxième quart du XII ${ }^{\mathrm{e}}$ siècle, est à quelques kilomètres de Tarascon, à Saint-Étienne-du-Grès, sur le tympan de la chapelle de Saint-Gabriel, où à côté du péché originel apparaît Daniel (préfiguration du Christ, le nouvel Adam) avec les lions (symbole fréquent de la luxure), opposition de scènes qui semble indiquer le sens sexuel du péché. Il est vrai qu'on a déjà dit que le contraste entre les deux scènes ne signifiait pas que l'artiste ait interprété le péché «comme un vulgaire péché de luxure, mais dans sa conséquence qui fut d'introduire le trouble et même la honte dans un domaine qui était sorti tout pur des mains du Créateur ${ }^{150} »$. Mais les auteurs de cette remarque semblent - phénomène longtemps courant dans les études d'art médiéval - enclins à adapter les intentions de l'artiste roman à la leçon théologiquement correcte, plutôt que d'envisager d'autres possibilités d'interprétation, extérieures au champ de la culture ecclésiastique. Il est significatif, par exemple, que sur le même espace du tympan les deux scènes soient chronologiquement inversées, d'abord celle de Daniel, ensuite celle du péché.

La deuxième image, italienne, figure sur la mosaïque d'Otrante (1163-1165). Les rameaux de l'arbre défendu y passent entre les jambes des personnages, insinuant la nature sexuelle du péché. Celle-ci semble d'autant plus évidente qu'Adam et Ève se trouvent chacun dans un cercle, ce qui fait des personnages des entités isolées, séparées, autonomes dans leurs domaines respectifs. Domaines résultant de la coupure en deux de l'androgyne primordial. Cette supposition est renforcée par le fait que le fruit défendu représenté ici est la figue (à la forte connotation sexuelle, on l'a déjà vu), figuré de façon suggestive par le mosaïste, le prêtre Pantaleone : la figue tenue par Ėve a sa partie plus mince tournée vers le bas et placée entre ses seins, comme si elle formait une troisième mamelle ; celle

150. Gérard de Champeaux et Sébastien Sterckx, Introduction au monde des symboles [1966], 3e éd., La Pierre-Qui-Vire, Zodiaque, 1980, p. 275. 
qui est dans la main d'Adam se trouve en position inverse et rappelle les parties génitales masculines ${ }^{151}$.

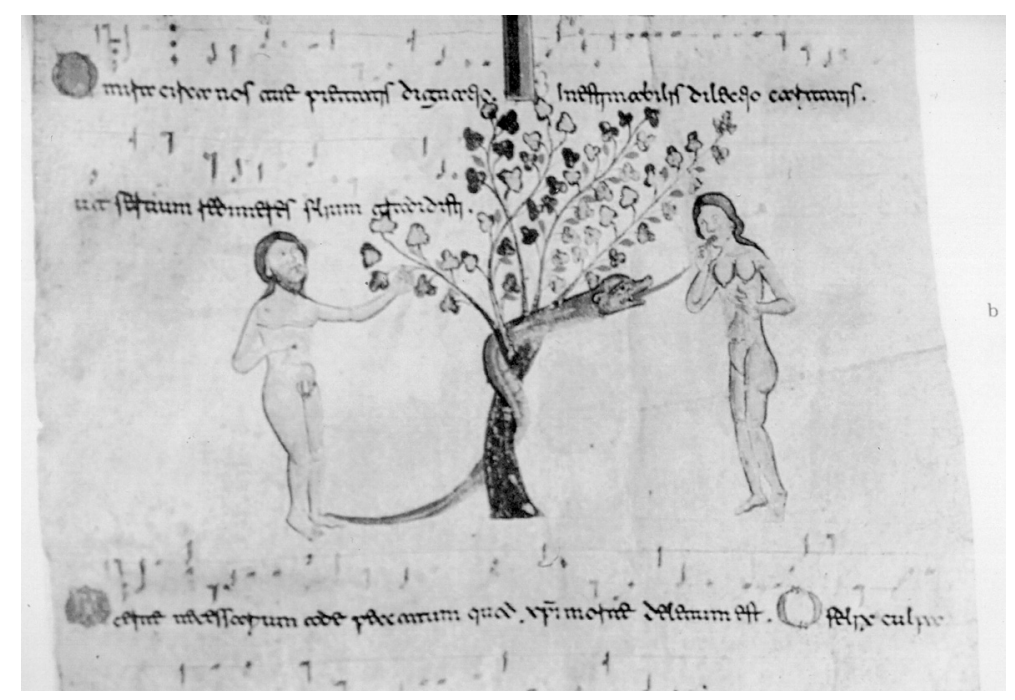

Figure 5. Enluminure de l'Exultet 3 de Troia (Pouille), Archivio Capitulario, milieu du $\mathrm{XI}^{\mathrm{e}}$ siècle.

Photo extraite de G. Cavallo, Rotoli di Exultet dell'Italia meridionale, Bari, Adriatico, 1973, pl. 54.

Prenant en compte la distribution géographique des images romanes, on voit que la fonction de fruit défendu attribuée à la figue s'exprimait surtout dans les milieux culturels liés au monde grécojudaïque, alors que la pomme apparaissait dans ceux liés au monde romano-chrétien. C'est peut-être grâce aux rapports particuliers que ces aires culturelles établissaient entre chacun de ces fruits et un organe, que, dans les images où la figue a été adoptée, Ève avec le fruit se trouve souvent du côté droit de l'arbre, comme le foie dans

151. Pareille attribution sexuelle était déjà apparue vers la fin du $\mathrm{X}^{\mathrm{e}}$ siècle en Espagne, dans un manuscrit du Beatus (Madrid, Bibliothèque de l'Escorial, Ms. 11.5, fol. 18). Il est vrai que cette représentation montre des feuilles de figuier à la place des fruits, mais celle qu'Adam porte a un caractère nettement phallique, et celle qui cache le sexe d'Eve est triangulaire comme une vulve. 


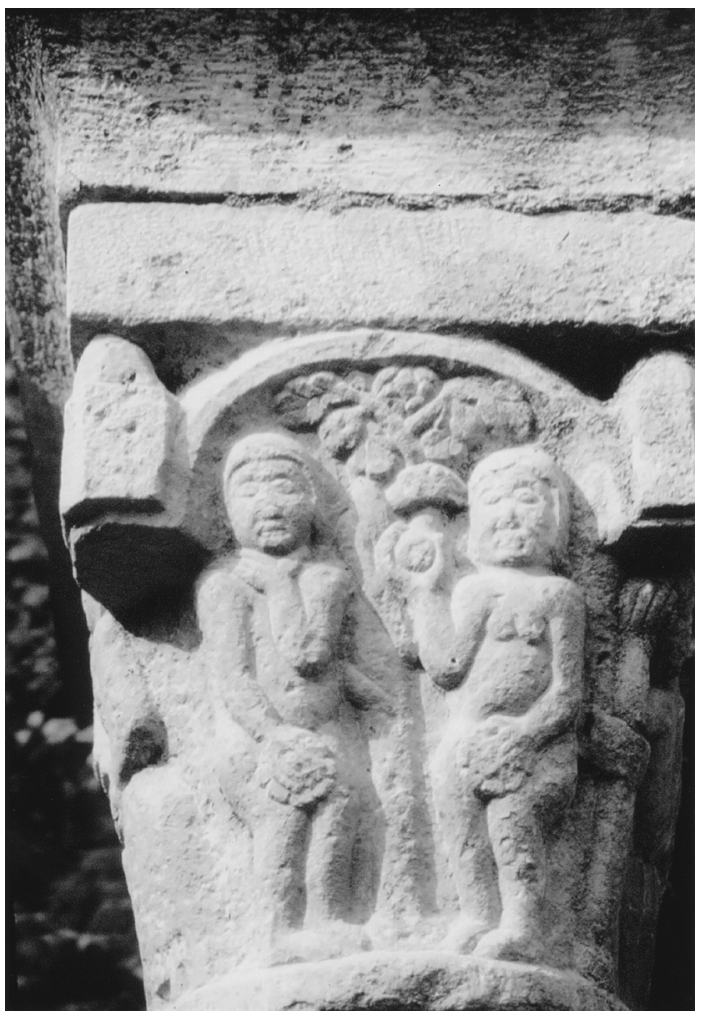

FiguRE 6. Chapiteau de la galerie ouest du cloître du monastère Santo Domingo, Peralada (Catalogne), XIII ${ }^{\mathrm{e}}$ siècle. Photo de l'auteur

le corps humain ${ }^{152}$. Dans les images où la pomme fut le choix, la tendance est qu'Ève et le fruit apparaissent à gauche, comme le cœur dans le corps humain (figures 3 et 6). Dans un cas comme dans l'autre, le fruit défendu était le symbole de la rupture de l'unité édénique, de la naissance de l'humanité disjointe qui caractérise l'Histoire.

Rua Julio Diniz 145, apart. 112

São Paulo 04547-090 BRÉSIL

152. À cet égard, nous parlons, évidemment, d'une tendance statistique, non d'une règle, puisque les cas contraires n'étaient pas rares, comme l'illustrent les figures 2 et 5 . 